UNIVERSIDADE FEDERAL DO PIAUÍ

COORDENAÇÃO EXECUTIVA DAS RESIDÊNCIAS MÉDICAS DA UFPI

$X$ JORNADA CIENTÍFICA DA

RESIDÊNCIA MÉDICA DA

UNIVERSIDADE FEDERAL DO PIAUÍ

ISSN: 2594-8687

12 A 15 DE FEVEREIRO 2019 AUDITÓRIO DO HU-UFPI TERRSINA PIAUÍ 

UNIVERSIDADE FEDERAL DO PIAUÍ

CENTRO DE CIÊNCIAS DA SAÚDE

COORDENAÇÃO EXECUTIVA DAS RESIDÊNCIAS MÉDICAS DA UFPI

\section{$X$ JORNADA CIENTÍFICA DA RESIDÊNCIA MÉDICA DA UNIVERSIDADE FEDERAL DO PIAUÍ}

12 A 15 FEVEREIRO - 2019 AUDITÓRIO DO HU/UFPI TERESINA - PIAUÍ 
FICHA CATALOGRÁFICA

Dados Internacional de Catalogação na Publicação (CIP) de acordo com ISBD

J828

Jornada Científia da Residência Médica da Universidade Federal do Piauí (10. :2019)

Anais da X Jornada da Residência Médica da

Universidade Federal do Piauí. - Teresina: EDUFPI, 2019. $42 \mathrm{p}$.

ISSN: 2594-8687

Hospital Universitário da Universidade Federal do Piauí HU - UFPI.

1. Hospital Universitário - Piauí. 2. Hospital Universitário - Evento. 3. Residência Médica. I. Titulo.

CDD 610.03

Elaborado por Marcelo Cunha de Andrade - Bibliotecário CRB/3 1221 
Reitor

Prof. Dr. José Arimatéia Dantas Lopes.

Vice-Reitora

Prof. ${ }^{a}$ Dr. $\stackrel{a}{ }$ Nadir do Nascimento Nogueira

Pró-Reitor de Ensino de Pós-Graduação

Prof. ${ }^{a}$ Dr. ${ }^{a}$ Regina Lucia Ferreira Gomes

Pró-Reitor de Pesquisa

Prof. Dr. João Xavier da Cruz Neto

Diretor do CCS

Prof. Dr. Viriato Campelo

Coordenadora Executivo da Residência Médica

Prof. ${ }^{a}$ Dr. ${ }^{a}$ Catarina Fernandes Pires 


\author{
Superintendente \\ José Miguel Luz Parente \\ Gerente de Ensino e Pesquisa
}

Marta Alves Rosal

Setor de Gestão da Pesquisa e Inovação Tecnológica

Maria do Carmo de Carvalho e Martins

Setor de Gestão do Ensino do HU-UFPI

Airton Mendes Conde Junior

Unidade Gerenciamento de atividades de Ensino de Pósgraduação do HU-UFPI

Murilo Moura Lima

Unidade de Gerenciamento de Atividades de Graduação e Ensino Técnico

Maria do Socorro Teixeira Moreira Almeida 


\section{Hospital Infantil Lucídio Portela}

Programa: Pediatria e Neonatologia

Diretor: Vinicius Pontes do Nascimento

Coord. COREME: Catarina Fernandes Pires

Hospital Areolino de Abreu

Programa: Psiquiatria

Diretor: Ralph Webster Cavalcante Trajano

Coord. COREME: Leonel Veloso Saraiva

\section{Maternidade Dona Evangelina Rosa}

Programa: Obstetrícia e Ginecologia

Diretor: Francisco de Macedo Neto

Coord. COREME: José Arimatéia dos Santos Júnior

Hospital Getúlio Vargas

Campo de Estágio

Diretora: Clara Francisca dos Santos Leal

Coord. COREME: Jozêlda Lemos Duarte

Hospital de Urgência de Teresina Dr. Zenon Rocha

Campo de Estágio

Diretor: Antonio Gillberto Albuquerque Brito 



\title{
X JORNADA DA RESIDÊNCIA MÉDICA DA UNIVERSIDADE FEDERAL DO PIAUÍ
}

Editoração e Organização

\author{
Marta Alves Rosal \\ Gerente de Ensino e Pesquisa
}

Murilo Moura Lima

Unidade Gerenciamento de atividades de Ensino de Pósgraduação do HU-UFPI

Marcelo Cunha de Andrade

Analista Administrativo - Biblioteconomia

Catarina Fernandes Pires

Coordenadora Executiva das Residências Médicas UFPI 


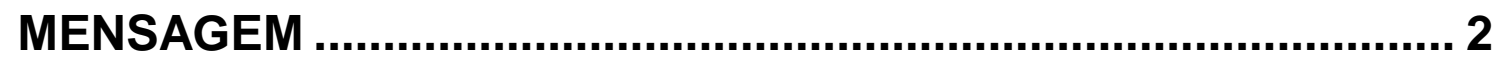

RESIDENTES CONCLUDENTES / PRM ...................................... 2

ORIENTADORES........................................................................ 2

PROGRAMAÇÃO CIENTÍFICA .................................................. 2

RESUMO DOS TRABALHOS DE CONCLUSÃO DO CURSO DE RESIDÊNCIA MÉDICA - 2019................................................. 2

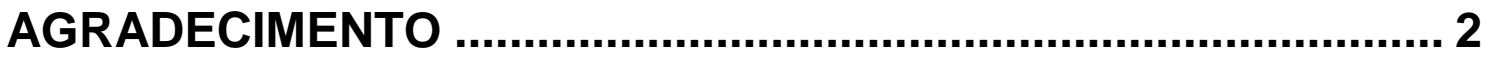




\section{MENSAGEM}

Caros Colegas,

A Jornada Científica da Residência Médica da Universidade Federal do Piauí é um dos principais eventos científicos da UFPI. Nessa Jornada, os médicos que estão concluindo seus programas de residência apresentarão pesquisas científicas de excelente qualidade, que retratam a excelência do ensino e da pesquisa médica na UFPI.

Neste evento estarão reunidos todos os médicos residentes, preceptores e supervisores dos diversos programas, compartilhando conhecimento médico e inovação em saúde.

Convidamos todos a participarem da X Jornada Científica da Residência Médica da UFPI e prestigiarem os novos profissionais especialistas oriundos da nossa Instituição.

Agradecemos a colaboração de todos que estiveram e continuam envolvidos com as Residências Médicas da UFPI.

Profa. Dra. Catarina Fernandes Pires

Coordenadora Executiva das Residências Médicas UFPI 
JOSÉ RODRIGO BEZERRA SOUSA

ATHANA DE OLIVEIRA CAVALCANTE

DENISE EVELYN MACHADO DE ALMEIDA

ANTONIO CHESLEM DE SOUZA

ANA PAULA DA SILVA SOARES

ISAAC ANTÃO DE CARVALHO NETO

VINY SAMPAIO DE BRITO

BETHANIA LUCIANA DOS SANTOS HOLANDA

GUILHERME SOARES BORGES

HERTON LUIZ ALVES SALES FILHO

LUIZ HENRIQUE CARVALHO DOS SANTOS

MARCONY BATISTA LIMA

MARIA DARISA DE SOUSA POLICARPO

PAULO HENRIQUE DE MELO FILHO

PRISCILA DA SILVEIRA SANTOS

ISADORA NOLETO BARBOSA

LAIZA CAVALCANTE DE CARVALHO CUNHA

LEONARDO ANTÔNIO CARVALHO HIPÓLITO

THÚLIO ADLEY LIMA CUNHA

LUIZA ÊNIA DA SILVA ARAÚJO

RENAN BASTOS LEITE

ALESSANDRA CARVALHO DE OLIVEIRA

CARLOS EDUARDO ALMEIDA SOUSA

FELIPE OSÓRIO MARQUES

LORENA NORBERTA MENDES MOURA

MARIA CLARA FORTES PORTELA BONFIM

THAINÁ PINTO DOS SANTOS

ANDRESSA DANIELA DE SOUSA

BEATRIZ DE OLIVEIRA FREIRE

DANIELE DE OLIVEIRA RODRIGUES

DANUSA DA PAZ OLIVEIRA DE SOUSA LEAL

JOSÉ HERMÍNIO ROCHA MAGALHÃES SANTOS

LAIS PORTELA NEIVA COELHO 
GUILHERME HENRIQUE MENDES LEAL DE SOUSA MARTINS

RAIMUNDO MOREIRA DOS SANTOS NETO

RICARDO COÊLHO FONTES

JORDANO SAMPAIO GUIMARÃES SILVA

ROBERTA SOARES GONDIM MEDEIROS FACUNDO 


\section{ORIENTADORES}

AILA DE MENEZES FERREIRA

ALEXANDRE CASTELO BRANCO VAZ PARENTE

ANA LÚCIA DA FRANÇA COSTA

ANA MARIA COÊLHO HOLANDA

ANAÍDE ROSA DE CARVALHO NASCIMENTO PINHEIRO

ANDRÉ GONÇALVES DA SILVA

ANTÔNIO DE DEUS FILHO

BRUNO PINHEIRO FALCÃO

CARLOS EDUARDO BATISTA DE LIMA

CAROLINE DE SOUSA COSTA

CATARINA FERNANDES PIRES

CLAUDIO HENRIQUE LIMA ROCHA

DÁYRTON RAULINO MOREIRA

DORCAS LAMOUNIER

EDNALDO ATEM GONÇALVES

EDUARDO BRUNO LOBATO MARTINS

EDUARDO HENRIQUE RAMOS DE SOUSA

GLENDA MARIA SANTOS MOREIRA

HORMONE OLIVEIRA RODRIGUES

IGOR DENIZARDE BACELAR MARQUES

JEANY BORGES E SILVA RIBEIRO

JOSÉ MARIA CORREIA LIMA E SILVA

LEONEL VELOSO SARAIVA

LUÍS GUSTAVO CAVALCANTE REINALDO

MARLON MORENO DA ROCHA CAMINHA DE PAULA

MARTA ALVES ROSAL

OSVALDO MENDES DE OLIVEIRA FILHO

PEDRO VITOR LOPES COSTA

RAIMUNDO ANTÔNIO CARDOSO JUNIOR

RAIMUNDO JOSÉ CUNHA ARAÚJO JUNIOR

SEMÍRAMIS JAMIL HADAD DO MONTE

SIMONE SOARES LIMA

VITOR GOMES PRADO 
DIA 12 DE FEVEREIRO DE 2019 (TERÇA-FEIRA)

LOCAL AUDITÓRIO DO HU-UFPI

$8 \mathrm{~h}$ às $9 \mathrm{~h}$ - SOLENIDADE DE ABERTURA

Autoridades:

Prof. Dr. José Arimatéia Dantas Lopes (Magnífico Reitor da UFPI)

Prof. Dr. José Miguel Luz Parente (Superintendente HU-UFPI)

Prof ${ }^{\mathrm{a}} \mathrm{Dr}^{\mathrm{a}}$ Regina Lucia Ferreira Gomes (Pró-Reitora de Ensino de Pós-Graduação da UFPI)

Prof. Dr. João Xavier Da Cruz Neto (Pró-Reitor de Pesquisa da UFPI)

Prof. Dr. Viriato Campelo (Diretor do Centro de Ciências da Saúde da UFPI)

Profa. Dra. Marta Alves Rosal (Gerente de Ensino e Pesquisa HUUFPI)

Prof. a Dr. a Catarina Fernandes Pires (Coordenadora Executiva da Residência Médica da UFPI)

9h às 10h - CONFERÊNCIA DE ABERTURA

Palestrante:

Prof ${ }^{a}$. Drạ. Lydia Masako Ferreira

10h às $10 \mathrm{~h} 30$ - COFFEE BREAK

DIA12 DE FEVEREIRO DE 2019 / TARDE

14h às $15 \mathrm{~h} 30$ - TCC DE ANESTESIOLOGIA E DERMATOLOGIA

TCC 01 - DETERMINANTES PARA SUSPENSÕES CIRÚRGICAS EM UM HOSPITAL UNIVERSITÁRIO

RESIDENTE: JOSÉ RODRIGO BEZERRA SOUSA

ORIENTADOR: EDUARDO HENRIQUE RAMOS DE SOUSA

PROGRAMA: ANESTESIOLOGIA

TCC 02 - QUALIDADE DE VIDA DE PACIENTES COM ACNE ATENDIDOS EM UM HOSPITAL UNIVERSITÁRIO

RESIDENTE: ATHANA DE OLIVEIRA CAVALCANTE

ORIENTADOR: CAROLINE DE SOUSA COSTA

PROGRAMA: DERMATOLOGIA

TCC 03 - PERFIL CLÍNICO E EPIDEMIOLÓGICO DOS PACIENTES PORTADORES DE PSORÍASE EM UM HOSPITAL UNIVERSITÁRIO

RESIDENTE: DENISE EVELYN MACHADO DE ALMEIDA

ORIENTADOR: ANA LÚCIA FRANÇA COSTA

PROGRAMA: DERMATOLOGIA 
TCC 04 - CORRELAÇÃO ENTRE ACHADOS ULTRASSONOGRÁFICOS E TRANSOPERATÓRIOS EM PACIENTES COM COLELITÍASE OPERADOS EM UM HOSPITAL UNIVERSITÁRIO

RESIDENTE: ANTONIO CHESLEM DE SOUZA

ORIENTADORA: LUÍS GUSTAVO CAVALCANTE REINALDO

PROGRAMA: CIRURGIA GERAL

TCC 05 - INFORMAÇÃO PRÉ-OPERATÓRIA DO PACIENTE E SATISFAÇÃO COM O ATENDIMENTO EM UMM HOSPITAL UNIVERSITÁRIO

RESIDENTE: ANA PAULA DA SILVA SOARES

ORIENTADOR: HORMONE OLIVEIRA RODRIGUES

PROGRAMA: CIRURGIA GERAL

TCC 06 - SULCO DE ROUVIERE: IDENTIFICAÇÃO E RECONHECIMENTO DESTE PONTO DE REFERÊNCIA NA COLECISTECTOMIAYVIDEOLAPAROSCÓPICA

RESIDENTE: ISAAC ANTÃO DE CARVALHO NETO

ORIENTADOR: RAIMUNDO JOSÉ CUNHA ARAÚJO JUNIOR

PROGRAMA: CIRURGIA GERAL

TCC 07 - PERFIL EPIDEMIOLÓGICO E DEMOGRÁFICO DOS PACIENTES SUBMETIDOS A CARDIOMIOPLASTIA VIDEOLAPAROSCÓPICA POR ACALÁSIA ESOFÁGICA NO HOSPITAL UNIVERSITÁRIO DA UNIVERSIDADE FEDERAL DO PIAUÍ DE JUNHO DE 2014 A JUNHO DE 2018

RESIDENTE: VINY SAMPAIO DE BRITO

ORIENTADOR: MARLON MORENO DA ROCHA CAMINHA DE PAULA

PROGRAMA: CIRURGIA GERAL

DIA 13 DE FEVEREIRO DE 2019 / MANHÃ

$8 \mathrm{~h}$ às 10h - TCC DE CLÍNICA MÉDICA

TCC 08 - PERFIL DOS PACIENTES COM TUMORES SÓLIDOS EM CUIDADOS PALIATIVOS INTERNADOS NO HOSPITAL UNIVERSITÁRIO DO PIAUÍ

RESIDENTE: BETHANIA LUCIANA DOS SANTOS HOLANDA

ORIENTADORES: CLAUDIO HENRIQUE LIMA ROCHA

PROGRAMA: CLIINICA MÉDICA

TCC 09 - PERFIL EPIDEMIOLÓGICO E CLÍNICO DOS PACIENTES SUBMETIDOS A BIÓPSIA RENAL NO HOSPITAL UNIVERSITÁRIO DA UNIVERSIDADE FEDERAL DO PIAUÍ (HU-UFPI)

RESIDENTE: GUILHERME SOARES BORGES

ORIENTADOR: IGOR DENIZARDE BACELAR MARQUES

PROGRAMA: CLIINICA MÉDICA 
TCC 10 - REATIVAÇÃO DE CITOMEGALOVÍRUS EM ADULTOS CRÍTICOS: ANÁLISE DE MORBIDADE E MORTALIDADE NA UTI DO HU-UFPI.

RESIDENTE: HERTON LUIZ ALVES SALES FILHO

ORIENTADOR: SEMÍRAMIS JAMIL HADAD DO MONTE

PROGRAMA: CLIINICA MÉDICA

TCC 11 - PERFIL EPIDEMIOLÓGICO DE PACIENTES SUBMETIDOS A BIÓPSIAS PULMONARES GUIADAS POR TOMOGRAFIA

RESIDENTE: LUIZ HENRIQUE CARVALHO DOS SANTOS

ORIENTADOR: ANTÔNIO DE DEUS FILHO

PROGRAMA: CLIINICA MÉDICA

DIA 13 DE FEVEREIRO DE 2019 / MANHÃ 10h às $11 \mathrm{~h} 30$ - TCC DE CLÍNICA MÉDICA

TCC 12 - PERFIL CLÍNICO EVOLUTIVO E TERAPÊUTICO DE PACIENTES COM MARCA-PASSO E CARDIODESFIBRILADOR IMPLANTÁVEL EM UM HOSPITAL UNIVERSITÁRIO

RESIDENTE: MARCONNY BATISTA LIMA

ORIENTADOR: CARLOS EDUARDO BATISTA DE LIMA

PROGRAMA: CLÍNICA MÉDICA

TCC 13 - LESÃO RENAL AGUDA EM PACIENTES SUBMETIDOS A CIRURGIA CARDÍACA

RESIDENTE: MARIA DARISA DE SOUSA POLICARPO

ORIENTADOR: RAIMUNDO ANTÔNIO CARDOSO JUNIOR

PROGRAMA: CLÍNICA MÉDICA

TCC 14 - AVALIAÇÃO DO CONTROLE GLICÊMICO DOS PACIENTES DIABÉTICOS TIPO 2 EM USO DE ANTIDIABÉTICOS ORAIS E INSULINAS

RESIDENTE: PAULO HENRIQUE DE MELO FILHO

ORIENTADOR: JOSÉ MARIA CORREIA LIMA E SILVA

PROGRAMA: CLIINICA MÉDICA

DIA 13 DE FEVEREIRO DE 2019 / TARDE

$14 \mathrm{~h}$ às $14 \mathrm{~h} 30$ - TCC DE ENDOSCOPIA DIGESTIVA, ENDOCRINOLOGIA E GERIATRIA

TCC 15 - MAPEAMENTO ENDOSCÓPICO DE ÚLCERAS GÁSTRICAS

RESIDENTE: PRISCILA DA SILVEIRA SANTOS

ORIENTADOR: JEANY BORGES E SILVA RIBEIRO

PROGRAMA: ENDOSCOPIA DIGESTIVA

TCC 16 - AVALIAÇÃO DOS NÍVEIS DE VITAMINA B12 EM PACIENTES DIABÉTICOS COM USO DE METFORMINA

RESIDENTE: ISADORA NOLETO BARBOSA

ORIENTADOR: ANDRÉ GONÇALVES DA SILVA

PROGRAMA: ENDOCRINOLOGIA E METABOLOGIA 
TCC 17 - EFEITOS DA MEDITAÇÃO GUIADA SOBRE O BEM-ESTAR FÍSICO E EMOCIONAL DE PACIENTES IDOSOS EM TRATAMENTO ONCOLÓGICO

RESIDENTE: LAIZA CAVALCANTE DE CARVALHO CUNHA

ORIENTADOR: GLENDA MARIA SANTOS MOREIRA

PROGRAMA: GERIATRIA

DIA 13 DE FEVEREIRO DE 2019 / TARDE

15h às 17h30 - TCC DE ORTOPEDIA E RADIOLOGIA E DIAG. POR IMAGEM

TCC 18 - FREQUÊNCIA DE SÍNDROME DO TÚNEL DO CARPO ASSOCIADA A DEDO EM GATILHO EM PACIENTES ATENDIDOS EM HOSPITAL UNIVERSITARIO DO PIAUÍ RESIDENTE: LEONARDO ANTONIO DE CARVALHO HIPÓLITO

ORIENTADOR: OSVALDO MENDES DE OLIVEIRA FILHO

PROGRAMA: ORTOPEDIA E TRAUMATOLOGIA

TCC 19 - TESTE DE PHALEN MODIFICADO NA SÍNDROME DO TÚNEL DO CARPO RESIDENTE: THÚLIO ADLEY LIMA CUNHA

ORIENTADOR: OSVALDO MENDES DE OLIVEIRA FILHO

PROGRAMA: ORTOPEDIA E TRAUMATOLOGIA

TCC 20 - CAPACIDADE PREDITIVA DO ESCORE DE WELLS NO DIAGNÓSTICO DE TROMBOSE VENOSA PROFUNDA RESIDENTE: LUIZA ÊNIA DA SILVA ARAÚJO

ORIENTADOR: EDUARDO BRUNO LOBATO MARTINS

PROGRAMA: RADIOLOGIA E DIAGNÓSTICO POR IMAGEM

TCC 21 - NEFROLITÍASE: COMPARAÇÃO ENTRE ULTRASSONOGRAFIA E TOMOGRAFIA COMPUTADORIZADA

RESIDENTE: RENAN BASTOS LEITE

ORIENTADOR: AILA DE MENEZES FERREIRA

PROGRAMA: RADIOLOGIA E DIAGNÓSTICO POR IMAGEM

DIA 14 DE FEVEREIRO DE 2019 / MANHÃ

8 às 11 h00 - TCC DE OBSTETRÍCIA E GINECOLOGIA

TCC 22 - ABORDAGEM DA SEXUALIDADE EM CONSULTAS AMBULATORIAIS POR GINECOLOGISTAS E OBSTETRAS

RESIDENTE: ALESSANDRA CARVALHO DE OLIVEIRA

ORIENTADOR: ANAÍDE ROSA DE CARVALHO NASCIMENTO PINHEIRO

PROGRAMA: OBSTETRÍCIA E GINECOLOGIA

TCC 23 - SATISFAÇÃO DAS PUÉRPERAS PÓS-PARTO VAGINAL EM MATERNIDADE DE REFERÊNCIA DO PIAUÍ

RESIDENTE: CARLOS EDUARDO ALMEIDA SOUSA 
ORIENTADOR: ANA MARIA COÊLHO HOLANDA

PROGRAMA: OBSTETRÍCIA E GINECOLOGIA

TCC 24 - ATIVIDADE SEXUAL E QUALIDADE DE VIDA DE MULHERES SUBMETIDAS A HISTERECTOMIA PARA TRATAMENTO DE LEIOMIOMA UTERINO

RESIDENTE: FELIPE OSÓRIO MARQUES

ORIENTADOR: ANAÍDE ROSA DE CARVALHO NASCIMENTO PINHEIRO

PROGRAMA: OBSTETRÍCIA E GINECOLOGIA

TCC 25 - FREQUÊNCIA E FATORES DE RISCO RELACIONADOS ÀS LESÕES PERINEAIS GRAVES EM PARTURIENTES

RESIDENTE: LORENA NORBERTA MENDES MOURA

ORIENTADOR: ANA MARIA COÊLHO HOLANDA

PROGRAMA: OBSTETRÍCIA E GINECOLOGIA

TCC 26 - FREQUÊNCIA DE FATORES AMBIENTAIS E COMPORTAMENTAIS ASSOCIADOS AO CÂNCER DE COLO UTERINO EM MULHERES ATENDIDAS EM HOSPITAL UNIVERSITÁRIO DO PIAUÍ

RESIDENTE: MARIA CLARA FORTES PORTELA BONFIM

ORIENTADOR: MARTA ALVES ROSAL

PROGRAMA: OBSTETRÍCIA E GINECOLOGIA

TCC 27 - FATORES PREDITORES DE INCONTINÊNCIA URINÁRIA CONFIRMADA EM ESTUDO URODINÂMICO EM MULHERES

RESIDENTE: THAINÁ PINTO DOS SANTOS

ORIENTADOR: PEDRO VITOR LOPES COSTA

PROGRAMA: OBSTETRÍCIA E GINECOLOGIA

14 DE FEVEREIRO DE 2019 / TARDE

$14 \mathrm{~h}$ às $17 \mathrm{~h}$ - TCC DE PEDIATRIA

TCC 28 - CONHECIMENTO DE CUIDADORES DE CRIANÇAS E ADOLESCENTES SOBRE SEGURANÇA NO TRÂNSITO

RESIDENTE: ANDRESSA DANIELA DE SOUSA

ORIENTADOR: SIMONE SOARES LIMA

PROGRAMA: PEDIATRIA

TCC 29 - RELAÇÃO ENTRE A TRANSMISSÃO VERTICAL DO HIV E AS CONDIÇÕES DA GRAVIDEZ E DO PARTO

RESIDENTE: BEATRIZ DE OLIVEIRA FREIRE

ORIENTADOR: DORCAS LAMOUNIER

PROGRAMA: PEDIATRIA

TCC 30 - CONHECIMENTO MATERNO SOBRE O TESTE DO PEZINHO EM MATERNIDADE DE REFERÊNCIA DO PIAUÍ

RESIDENTE: DANIELE DE OLIVEIRA RODRIGUES

ORIENTADOR: DÁYRTON RAULINO MOREIRA

PROGRAMA: PEDIATRIA 
TCC 31 - CONHECIMENTO DOS CUIDADORES SOBRE AS PATOLOGIAS DE CRIANÇAS E ADOLESCENTES INTERNADOS E OS FATORES ASSOCIADOS

RESIDENTE: DANUSA DA PAZ OLIVEIRA DE SOUSA LEAL

ORIENTADOR: SIMONE SOARES LIMA

PROGRAMA: PEDIATRIA

TCC 32 - PERFIL DE ADOLESCENTES COM COMPORTAMENTOS AUTOLESIVOS EM ESCOLA PÚBLICA DE CAXIAS - MARANHÃO

RESIDENTE: JOSÉ HERMÍNIO ROCHA MAGALHÃES SANTOS

ORIENTADOR: CATARINA FERNANDES PIRES

PROGRAMA: PEDIATRIA

TCC 33 - RELAÇÃO ENTRE TEMPO DE PERMANÊNCIA HOSPITALAR E BACTÉRIAS MULTIDROGA RESISTENTES EM HOSPITAL INFANTIL DE REFERÊNCIA

RESIDENTE: LAIS PORTELA NEIVA COELHO

ORIENTADOR: BRUNO PINHEIRO FALCÃO

PROGRAMA: PEDIATRIA

15 DE FEVEREIRO DE 2019 / MANHÃ

$8 \mathrm{~h}$ às $9 \mathrm{~h} 30$ - OFTALMOLOGIA

TCC 34 - CUSTO DO TRATAMENTO MEDICAMENTOSO DO GLAUCOMA PARA PACIENTES ATENDIDOS EM UM AMBULATÓRIO PÚBLICO

RESIDENTE: GUILHERME HENRIQUE MENDES LEAL DE SOUSA MARTINS

ORIENTADOR: VITOR GOMES PRADO

PROGRAMA: OFTALMOLOGIA

TCC 35 - ALTERAÇÕES OFTALMOLÓGICAS EM PORTADORES DE DOENÇA FALCIFORME EM HOSPITAL DE TERESINA

RESIDENTE: RAIMUNDO MOREIRA DOS SANTOS NETO

ORIENTADOR: EDNALDO ATEM GONÇALVES E GILDENE ALVES DA COSTA

PROGRAMA: OFTALMOLOGIA

TCC 36 - ACHADOS CLíNICOS E PATOLÓGICOS DE RETINOBLASTOMA EM HOSPITAL DE TERESINA, PIAUÍ

RESIDENTE: RICARDO COÊLHO FONTES

ORIENTADOR: EDNALDO ATEM GONÇALVES

PROGRAMA: OFTALMOLOGIA

15 DE FEVEREIRO DE 2019 / TARDE

$9 \mathrm{~h} 30$ às $10 \mathrm{~h} 30$ - PSIQUIATRIA

TCC 37 - IDEAÇÃO SUICIDA E FATORES ASSOCIADOS EM ESTUDANTES DE MEDICINA 
RESIDENTE: JORDANO SAMPAIO GUIMARÃES SILVA

ORIENTADOR: ALEXANDRE CASTELO BRANCO VAZ PARENTE

PROGRAMA: PSQUIATRIA

TCC 38 - USO DE SUBSTANCIAS PSICOATIVAS EM ACADÊMICOS DE MEDICINA RESIDENTE: ROBERTA SOARES GONDIM MEDEIROS FACUNDO

ORIENTADOR: LEONEL VELOSO SARAIVA

PROGRAMA: PSIQUIATRIA

ENCERRAMENTO E DIVULGAÇÃO DOS MELHORES TRABALHOS- 10h30 - 12h. 
TCC 01 - DETERMINANTES PARA SUSPENSÕES CIRÚRGICAS EM UM HOSPITAL UNIVERSITÁRIO

RESIDENTE: JOSÉ RODRIGO BEZERRA SOUSA

PROGRAMA: ANESTESIOLOGIA

ORIENTADOR: EDUARDO HENRIQUE RAMOS DE SOUSA

INTRODUÇÃO: A taxa de suspensão de cirurgias é um indicador da qualidade de atendimento e gestão oferecida pela instituição. A análise desta taxa visa a melhoria da qualidade da assistência e da eficiência do serviço. OBJETIVOS: Calcular a taxa de suspensão de cirurgias no Hospital Universitário da Universidade Federal do Piauí (HUUFPI) em um período de 12 meses. Caracterizar as principais causas de cancelamento e especificar a taxa de suspensão geral e por especialidade cirúrgica, bem como por porte cirúrgico. METODOLOGIA: Trata-se de um estudo transversal de natureza descritiva. Os dados coletados são referentes às cirurgias agendadas no período 01 de janeiro de 2017 a 31 de dezembro de 2017. Foi calculada a taxa de suspensão cirúrgica geral, por especialidades e portes cirúrgicos, bem como os motivos de suspensão. RESULTADOS: Durante o período estudado foram agendados 5008 procedimentos cirúrgicos. Destes, 4229 foram realizados e 779 foram cancelados, resultando numa taxa de cancelamento geral de $15,55 \%$. A especialidade cirúrgica com maior índice de cancelamento foi a Cirurgia Cardiovascular. A maioria das cirurgias suspensas foram de pequeno porte. Os principais motivos de cancelamento estão associados ao paciente, sendo estes os principais motivos o absenteísmo do paciente e a falta de condições clínica. Dentre as condições clínicas do paciente se destaca a hipertensão e o fato que $22,70 \%$ dos cancelamentos por esse motivo não tiveram detalhes clínicos revelados. CONCLUSÃO: A taxa geral de cancelamento foi de $15,55 \%$. A especialidade com maior taxa de suspensão foi a Cirurgia Cardiovascular. Os principais motivos de cancelamento foram relacionados ao paciente.

PALAVRAS-CHAVE: Liberação de Cirurgia. Centro Cirúrgico Hospitalar. Indicadores de Qualidade em Assistência à Saúde.

TCC 02 - QUALIDADE DE VIDA DE PACIENTES COM ACNE ATENDIDOS EM UM HOSPITAL UNIVERSITÁRIO

RESIDENTE: ATHANA DE OLIVEIRA CAVALCANTE

PROGRAMA: DERMATOLOGIA

INSTITUIÇÃO: HOSPITAL UNIVERSITÁRIO DA UNIVERSIDADE FEDERAL DO PIAUÍ

ORIENTADOR: CAROLINE DE SOUSA COSTA E PAULO CÉSAR DOS SANTOS

INTRODUÇÃO: A acne é frequentemente entendida como uma dermatose simples e autolimitada; porém, pode ser relacionada a transtornos psicológicos graves. OBJETIVO: O presente estudo objetiva mensurar o impacto provocado pela acne na qualidade de vida dos pacientes atendidos nos ambulatórios de Dermatologia do Hospital Universitário da Universidade Federal do Piauí (HU-UFPI). METODOLOGIA. Um estudo transversal foi realizado no período entre março e setembro de 2018. A amostra por conveniência incluiu 96 pacientes. Todos assinaram um termo de consentimento livre e esclarecido. 0 questionário Cardiff acne disability index (CADI), traduzido e validado para uso no Brasil, 
foi utilizado. RESULTADOS: O escore CADI médio foi de 5,5. Observou-se um predomínio de pacientes do sexo feminino $(75 / 78,1 \%)$, solteiros $(n=82 / 85,5 \%)$, não inseridos no mercado de trabalho ( $n=67 / 69,8 \%$ ) com segundo grau completo $(n=64 / 66,7 \%)$ e residentes na zona urbana $(n=81 / 84,4 \%)$. Conforme a classificação clínica, pacientes com acne moderada $(n=41 / 42,7 \%$ ) e cicatrizes ( $n=64 / 66,7 \%$ foram predominantes. Não foi encontrado associação estatisticamente significante entre as variáveis analisadas e o comprometimento da qualidade de vida dos pacientes atendidos. CONCLUSÃO: O comprometimento da qualidade de vida dos pacientes com acne atendidos no HU-UFPI foi moderado e não houve associação estatisticamente significante com as variáveis estudadas. A percepção da doença pelo próprio paciente e o estresse psicossocial relacionado devem ser as considerações mais importantes para a instituição e escolha do tratamento.

PALAVRAS-CHAVE: Acne. Dermatologia. Qualidade de Vida.

TCC 03 - PERFIL CLÍNICO E EPIDEMIOLÓGICO DOS PACIENTES PORTADORES DE PSORÍASE EM UM HOSPITAL UNIVERSITÁRIO

\section{RESIDENTE: DENISE EVELYN MACHADO DE ALMEIDA \\ PROGRAMA: DERMATOLOGIA \\ INSTITUIÇÃO: HOSPITAL UNIVERSITÁRIO DA UNIVERSIDADE FEDERAL DO PIAUÍ ORIENTADOR: ANA LÚCIA FRANÇA COSTA}

INTRODUÇÃO: Psoríase é doença inflamatória crônica da pele, unhas e articulações, recorrente, imunomediada, de base genética, com grande polimorfismo de expressão clínica. De caráter universal, ocorre como placas eritematodescamativas, com interferência expressiva na qualidade de vida dos indivíduos acometidos. OBJETIVO: Avaliar as características clínicas e epidemiológicas dos pacientes portadores de Psoríase que fazem seguimento no ambulatório de dermatologia, no Hospital Universitário da Universidade Federal do Piauí. METODOS: Foi realizado um estudo transversal, observacional, descritivo em que foram aplicados questionários aos pacientes portadores de Psoríase que realizaram acompanhamento no ambulatório de Dermatologia do HUUFPI, nos meses de abril de 2018 a agosto de 2018. RESULTADOS: Houve predomínio de pacientes do sexo masculino, casados, pardos, com baixo nível de instrução, renda de pouco mais de um salário mínimo, provenientes da zona urbana do interior do Piauí, com idade média de 47 anos. As principais comorbidades associadas foram hipertensão arterial, dislipidemia e diabetes melitus tipo 2. Artrite psoriásica foi associada em um terço dos casos. Tratamento tópico com clobetasol foi realizado em $40 \%$ dos pacientes e quase um terço dos pacientes faziam uso do imunossupressor metotrexato, enquanto $20 \%$ dos pacientes estavam em uso de imunobiológicos. Não é realizado tratamento com fototerapia neste hospital. CONCLUSÃO: O perfil encontrado encontra-se em consonância com dados da literatura mundial, que relatam o predomínio de casos graves em pacientes do sexo masculino, bem como associação com doenças que fazem parte da Síndrome Metabólica e com artrite psoriásica. O tratamento fototerápico poderia ser implementado.

PALAVRAS-CHAVE: Doenças cutâneas. Psoríase. Epidemiologia. 
TCC 04 - CORRELAÇÃO ENTRE ACHADOS ULTRASSONOGRÁFICOS E TRANSOPERATÓRIOS EM PACIENTES COM COLELITÍASE OPERADOS EM UM HOSPITAL UNIVERSITÁRIO

\author{
RESIDENTE: ANTONIO CHESLEM DE SOUZA \\ PROGRAMA: CIRURGIA GERAL \\ ORIENTADOR: LUIS GUSTAVO CAVALCANTE REINALDO
}

INTRODUÇÃO: Na colelitíase, a ultrassonografia é importante tanto para o diagnóstico como para planejamento operatório. OBJETIVOS: Descrever variáveis da vesícula biliar (VB) e vias biliares constantes nos laudos ultrassonográficos e compará-las com os achados operatórios nas colecistectomias videolaparoscópicas. METODOLOGIA: Estudo transversal, observacional, descritivo. Foram analisados 83 pacientes portadores de colelitíase submetidos à colecistectomia videolaparoscópica e que tinham ultrassonografia prévia. Foram investigadas variáveis ultrassonográficas da VB e vias biliares e comparadas com achados intraoperatórios. RESULTADOS: Frequência de aferição das variáveis ultrassonográficas: presença de cálculos biliares - 100\%; distensão da VB 44,6\%; espessamento parietal da VB - 59\%; lama biliar - 60,2\%; dilatação das vias biliares 60,2\%; grau de dilatação do colédoco $60,2 \%$ e topografia da VB $60,2 \%$. Comparação entre achados ultrassonográficos e intraoperatórios: distensão da VB $-p$ valor 0,211 ; espessamento da parede da VB $-p$ valor 1 ; presença de cálculos biliares $-p$ valor 1. A ultrassonografia apresentou para a variável distensão da VB acurácia de $56,8 \%$, sensibilidade $64,3 \%$, especificidade de $52,2 \%$, VPP $45 \%$ e VPN $70,6 \%$; para espessura da parede da VB acurácia $71,4 \%$, sensibilidade $66,7 \%$, especificidade $75 \%$, VPP $66,7 \%$ e VPN 75\%; para a variável presença de cálculo obteve-se sensibilidade de $100 \%$ e VPP de $100 \%$. CONCLUSÃO: Parcela significativa dos laudos não descreveram variáveis ultrassonográficas importantes. Não houve diferenças estatísticas significativas entre os achados ultrassonográficos e intraoperatórios, sendo maior concordância obtida para a variável presença de cálculos biliares.

PALAVRAS-CHAVE: Colelitíase. Ultrassonografia. Colecistectomia.

TCC 05 - INFORMAÇÃO PRÉ-OPERATÓRIA DO PACIENTE E SATISFAÇÃO COM O ATENDIMENTO EM UMM HOSPITAL UNIVERSITÁRIO

RESIDENTE: ANA PAULA DA SILVA SOARES

PROGRAMA: CIRURGIA GERAL

ORIENTADOR: HORMONE OLIVEIRA RODRIGUES

INTRODUÇÃO: A Resposta Endócrino-Metabólica-Imunológica ao Trauma cirúrgico é influenciada por fatores benéficos e maléficos, antes, durante e após a cirurgia. O ERAS society (Enhanced Recovery After Surgery), estabeleceu orientações perioperatórias com as melhores evidências científicas, para controlar estes fatores e acelerar a recuperação pós-operatória, incentivando a criação de diversos protocolos semelhantes, como o ACERTO (Acelerando a Recuperação Total Pós-operatória), que inicialmente voltado para cirurgias colorretais, atualmente é utilizado como guia para orientação cirúrgica em geral. Dentre suas diretrizes, está o incentivo à informação pré-operatória adequada. OBJETIVO: identificar falhas na informação pré-operatória de pacientes eletivos da Cirurgia Geral no Hospital Universitário da UFPI para posteriormente encontrar meios de melhorá-la e conhecer a satisfação dos pacientes. MÉTODOS: Estudo observacional descritivo, amostragem não probabilística, seleção dos pacientes por conveniência. População: pacientes internados em dezembro/2018 para realizar herniorrafia umbilical, herniorrafia inguinal ou colecistectomia videolaparoscópica eletivas. Coleta de dados: aplicação de questionários no pré e pós-operatório. RESULTADOS: Dos 22 pacientes, 
$81,8 \%$ eram do sexo feminino, $59,1 \%$ do interior do Piauí, $50 \%$ residentes em Teresina; com idade média $47 \pm 17,84$ anos; $9,1 \%$ não sabiam que cirurgia e $27,3 \%$ não sabiam que anestesia seriam realizadas; $40,9 \%$ desconheciam termo de consentimento, $40 \%$ desconheciam motivo do jejum, 59,1\% desconheciam complicações; $66,6 \%$ desconheciam previsão de alta; $77,3 \%$ gostariam de receber material explicativo sobre pré e $81 \%$ sobre pós-operatório. CONCLUSÃO: Foram identificadas falhas na informação pré-operatória e conhecida a satisfação dos pacientes com o atendimento. É necessário informar melhor os pacientes sobre doença, tratamento, consentimento informado e detalhes da internação.

PALAVRAS-CHAVE: Herniorrafia. Colecistectomia Videolaparoscópica. Satisfação do paciente. Consentimento Livre e Esclarecido.

TCC 06 - SULCO DE ROUVIERE: IDENTIFICACÃO E RECONHECIMENTO DESTE PONTO DE REFERÊNCIA NA COLECISTECTOMIAVIDEOLAPAROSCÓPICA

RESIDENTE: ISAAC ANTÃO DE CARVALHO NETO

PROGRAMA: CIRURGIA GERAL

ORIENTADOR: RAIMUNDO JOSÉ CUNHA ARAÚJO JÚNIOR

INTRODUÇÃO: Com o avanço da colecistectomiavideolaparoscópica para tratamento de cálculos de vesícula biliar, discutiram-se estratégias para a realização de cirurgias de forma segura, buscando reduzir complicações como: lesão de ducto biliar e sangramento. A identificação do sulco de Rouviere pode ajudar a evitar lesões, dentre elas a lesão do ducto colédoco durante a colecistectomia laparoscópica, pois é um indicador do local apropriado para iniciar a dissecação do triângulo Calot. OBJETIVOS: Avaliar a identificação do sulco de Rouviere em pacientes submetidos à colecistectomia laparoscópica, na unidade de cirurgia do Hospital Universitário da Universidade Federal do Piauí (HU-UFPI), no período de 10 de outubro a 26 de dezembro de 2018. METODOLOGIA: Trata-se de um estudo transversal, observacional, quantitativo. Será feita uma análise descritiva através das frequências absolutas (№) e relativas (\%) e a prevalência do sulco de Rouviere na população estudada. RESULTADOS: Foram analisados 92 pacientes, dos quais 82 apresentavam a presença do Sulco de Rouviere, que corresponde a $89,1 \%$ dos pacientes, e $10,9 \%$ dos pacientes não apresentavam. Dos 82 pacientes que apresentaram a presença, $68(82,9 \%)$ pacientes apresentaram na forma de sulco real, e $14(17,1 \%)$ pacientes apresentaram na forma de cicatriz. $53(64,6 \%)$ pacientes apresentaram o Sulco de Rouviére na direção oblíqua, 26 (31,7\%) na direção horizontal e apenas $3(3,7 \%)$ na vertical. CONCLUSÃO: A pesquisa demonstrou que o sulco de Rouviere é um marco anatômico que pode ser reconhecido na colecistectomiavideolaparoscópica, sendo a maioria sob a forma de sulco real ou fosseta.

PALAVRAS-CHAVE: Colecistectomiavideolaparoscópica. Sulco de Rouviere. Lesãoiatrogênica da Via Biliar.

TCC 07 - PERFIL EPIDEMIOLÓGICO E DEMOGRÁFICO DOS PACIENTES SUBMETIDOS A CARDIOMIOPLASTIA VIDEOLAPAROSCÓPICA POR ACALÁSIA ESOFÁGICA NO HOSPITAL UNIVERSITÁRIO DA UNIVERSIDADE FEDERAL DO PIAUÍ DE JUNHO DE 2014 A JUNHO DE 2018

RESIDENTE: VINY SAMPAIO DE BRITO

PROGRAMA: CIRURGIA GERAL

INSTITUIÇÃO: HOSPITAL UNIVERSITÁRIO DA UNIVERSIDADE FEDERAL DO PIAUÍ ORIENTADOR: MARLON MORENO DA ROCHA CAMINHA DE PAULA 
INTRODUÇÃO: Acalásia esofágica é rara, sua incidência é maior em países com maior prevalência da doença de chagas. Fisiopatologia compreende a destruição dos plexos mioentéricos esofágicos que levam a não dilatação fisiológica do esfíncter esofagiano inferior. Há morbidade importante com comprometimento da qualidade de vida e inanição. No Piauí não há estudos sobre a situação da acalásia nem sobre seu tratamento. OBJETIVOS:. Determinar e avaliar idade, sexo, naturalidade e procedência, peso e disfagia pré e pós-cirúrgica dos pacientes diagnosticados com acalasia esofágica e submetidos a cardiomioplastia videolaparoscópica no HU-UFPI no período de Junho de 2014 a Junho de 2018. METODOLOGIA: Estudo retrospectivo, observacional e quantitativo. As variáveis consideradas foram sexo, idade, naturalidade e procedência, índice de massa corpórea, disfagia pré e pós-cirúrgica. Os dados foram analisados no software $R$ versão 3.5.1. A amostra foi caracterizada por meio de frequências absolutas e relativas percentuais, também por meio de estatística descritivas: mínimo, máximo, média e desvio padrão. RESULTADOS: Foram analisados 22 pacientes, sendo $54,55 \%$ de pacientes do sexo masculino, 90,91\% naturais do Piauí, 72,73\% procedentes do interior do estado, 4,55\% submetidos a tratamento prévio, $50 \%$ portador de Megaesôfago Grau 2 de Rezende. $81,82 \%$ sem disfagia pós-operatória, $68,18 \%$ não realizaram sorologia para chagas, e $22,73 \%$ de sorologias vieram negativas. IMC pré-operatório médio de 23,93. CONCLUSÃO: Os pacientes apresentam características epidemiológicas e demográficas compatíveis com a literatura nacional e internacional necessitando de mais estudos para melhor aprofundamento no entendimento da situação da doença no estado.

PALAVRAS-CHAVE: Acalásia. Esôfago. Cardiomiotomia Videolaparoscópica.

TCC 08 - PERFIL DOS PACIENTES COM TUMORES SÓLIDOS EM CUIDADOS PALIATIVOS INTERNADOS NO HOSPITAL UNIVERSITÁRIO DO PIAUÍ

RESIDENTE: BETHANIA LUCIANA DOS SANTOS HOLANDA

PROGRAMA: CLÍNICA MÉDICA

INSTITUIÇÃO: HOSPITAL UNIVERSITÁRIO DA UNIVERSIDADE FEDERAL DO PIAUÍ

ORIENTADOR: CLAUDIO HENRIQUE LIMA ROCHA E SÁVIA RAQUEL COSTA NORMANDO

INTRODUÇÃO: O câncer é atualmente a segunda causa de morte no Brasil e no mundo. Esta doença causa maior impacto em países em desenvolvimento, pois cerca de $80 \%$ dos pacientes são diagnosticados com câncer em fases avançadas da doença. Nestes casos, a terapia paliativa está indicada. OBJETIVO: Analisar o perfil clínico-epidemiológico dos pacientes com tumores sólidos em cuidados paliativos internados no Hospital Universitário do Piauí. METODOLOGIA: Estudo descritivo e transversal, realizado na enfermaria de clínica médica e oncologia do Hospital Universitário do Piauí no período de julho a novembro de 2018. As variáveis analisadas foram gênero, idade, procedência, nível de escolaridade, renda familiar, tipo de câncer, estadiamento, tratamento utilizado, causas de internações. A amostra foi caracterizada por meio de frequências absolutas e relativas, média e desvio padrão. RESULTADOS: Foram avaliados 63 pacientes, com idade média de 59,2 anos, sendo $63,5 \%$ do gênero feminino. O câncer do colo do útero foi o mais comum no gênero feminino $(25 \%)$, enquanto o câncer de pulmão $(26,1 \%)$ foi o mais frequente no gênero masculino. Dos pacientes estudados, $85,2 \%$ possuíam estadiamento IV. As complicações infecciosas ocorreram em $46,6 \%$ dos pacientes. Em relação ao tratamento, a quimioterapia e cuidados paliativos exclusivos foram utilizados por $40,3 \%$ e $29 \%$, respectivamente. CONCLUSÃO: Os dados obtidos permitiram caracterizar o perfil clinico e epidemiológico dos pacientes em cuidados paliativos, servindo como base de dados para estudos epidemiológicos futuros e como subsídio para 
a implementação de serviço especializado em Cuidados Paliativos no Hospital Universitário do Piauí.

PALAVRAS-CHAVE: Câncer, Cuidados Paliativos, Hospital Universitário do Piauí.

TCC 09 - PERFIL EPIDEMIOLÓGICO E CLÍNICO DOS PACIENTES SUBMETIDOS A BIÓPSIA RENAL NO HOSPITAL UNIVERSITÁRIO DA UNIVERSIDADE FEDERAL DO PIAUÍ (HU-UFPI)

RESIDENTE: GUILHERME SOARES BORGES

PROGRAMA: CLÍNICA MÉDICA

INSTITUIÇÃO: HOSPITAL UNIVERSITÁRIO DA UNIVERSIDADE FEDERAL DO PIAUÍ ORIENTADOR: IGOR DENIZARDE BACELAR MARQUES

INTRODUÇÃO: A biópsia renal foi introduzida em 1951, tendo a histologia renal começado a ser avaliada posteriormente, e a ultrassonografia associada ao procedimento na década de 80. É fator positivo na definição precoce de patologias, sejam elas primárias ou secundárias, além do estabelecimento de um banco de dados das principais patologias renais. OBJETIVOS: Caracterizar epidemiologicamente os pacientes submetidos à biópsia renal, além de definir os principais fatores indicativos, complicações pós procedimento e achados histológicos relacionados à biópsia renal no HU-UFPI. METODOLOGIA: Estudo retrospectivo baseado na coleta de dados em prontuário dos pacientes submetidos a biópsia renal no período entre janeiro de 2015 e outubro de 2018. As variáveis consideradas foram sexo, idade, raça, perfil de comorbidades, perfil laboratorial pré-biópsia, histologia e fatores indicativos para a mesma. Os dados foram analisados no software SPSS®, versão 20.0. A amostra foi caracterizada por frequências absolutas e percentuais, média e desvio padrão. RESULTADOS: Analisados 128 pacientes, 123 aptos a participar da pesquisa, $60 \%$ do sexo feminino, com idade média de 36 anos. Síndrome nefrótica e comprometimento renal sistêmico foram as principais indicações, totalizando 65\%. Dois terços das biópsias demonstraram glomerulopatias secundárias (Nefrite lúpica 76\%) e 29\% primárias (GESF 39\%). Entre as classes das nefrites lúpicas, a classe IV foi predominante, representando 62\%. CONCLUSÃO: Assim como no restante do Brasil, a principal glomerulopatia primária diagnosticada foi a glomeruloesclerose segmentar e focal. Com relação às glomerulopatias secundárias, a nefrite lúpica foi a mais prevalente. O procedimento de biópsia renal por US mostrou-se seguro com apenas 3\% de complicações.

PALAVRAS-CHAVE: Biópsia Renal. Glomerulopatia. Nefrite Lúpica.

TCC 10 - REATIVAÇÃO DE CITOMEGALOVÍRUS EM ADULTOS CRÍTICOS: ANÁLISE DE MORBIDADE E MORTALIDADE NA UTI DO HU-UFPI.

\section{RESIDENTE: HERTON LUIZ ALVES SALES FILHO}

PROGRAMA: CLÍNICA MÉDICA

INSTITUIÇÃO: HOSPITAL UNIVERSITÁRIO DA UNIVERSIDADE FEDERAL DO PIAUÍ ORIENTADOR: SEMIRAMIS JAMIL HADAD DO MONTE

INTRODUÇÃO: Citomegalovírus é um vírus altamente patogênico na vida intrauterina e em pacientes imunocomprometidos. Pesquisas das últimas 3 décadas apontam esse vírus como um agente que piora a mortalidade e morbidade em pacientes adultos imunocompetentes admitidos na UTI. OBJETIVO: Mensurar o impacto da morbidade e mortalidade da reativação do CMV entre os adultos críticos imunocompetentes atendidos na UTI do HU UFPI. MÉTODOS: Dentro dos pacientes aptos a participar do estudo, foi 
colhida amostra de sangue no quinto dia de admissão na UTI para mensuração da carga viral e separação dos grupos de acordo com a positividade no teste. As variáveis avaliadas foram tempo de estadia hospitalar, tempo de estadia na UTI e desfecho clínico (alta versus óbito). Os dados foram analisados usando o software $R$ versão 3.5.1. RESULTADOS: Foram incluídos no estudo 18 pacientes. Desses, $4(22,2 \%)$ tiveram a viremia por CMV reativada no quinto dia. Todos os pacientes com CMV foram a óbito, e somente 5 dos 14 pacientes sem CMV foram a óbito - entretanto, não houve diferença estatística entre os grupos no teste exato de Fisher $(p=0,082)$. O tempo de internação na UTI e o tempo de internação hospitalar foram semelhantes entre os pacientes com e sem reativação de CMV no presente estudo. CONCLUSÃO: a reativação de CMV não mostrou impacto significativo na morbimortalidade na população estudada.

Palavras-chave: Citomegalovírus. Cuidados Críticos. Mortalidade. Morbidade.

TCC 11 - PERFIL EPIDEMIOLÓGICO DE PACIENTES SUBMETIDOS A BIÓPSIAS PULMONARES GUIADAS POR TOMOGRAFIA COMPUTADORIZADA EM UM HOSPITAL UNIVERSITÁRIO DO PIAUÍ

\section{RESIDENTE: LUIZ HENRIQUE CARVALHO DOS SANTOS \\ PROGRAMA: CLÍNICA MÉDICA \\ INSTITUIÇÃO: HOSPITAL UNIVERSITÁRIO DA UNIVERSIDADE FEDERAL DO PIAUÍ ORIENTADOR: ANTÔNIO DE DEUS FILHO}

Introdução: A investigação histológica dos nódulos e massas pulmonares é muito importante para se obter o diagnóstico diferencial entre lesões malignas e benignas, tendo em vista que a neoplasia pulmonar é um dos cânceres mais frequentes no nosso meio, relacionado diretamente ao tabagismo, necessitando de um diagnóstico rápido e preciso, e a biópsia pulmonar percutânea guiada por tomografia computadorizada (BPPTC) é um exame com boa sensibilidade e acurácia, pouco invasivo e de poucas complicações, de fundamental importância para os diagnósticos histopatológicos. Objetivos: Traçar o perfil epidemiológico dos pacientes em investigação de nódulos pulmonares no Hospital Universitário da UFPI através da BPPTC, descrevendo ainda as complicações do procedimento e os resultados histopatológicos do exame. Metodologia: Estudo descritivo, observacional e retrospectivo. Através da análise de prontuários e do registro de BPPTC. Foram excluídos do diagnóstico histopatológico final, os pacientes que perderam segmento. Os dados foram registrados em planilhas do Microsoft Excel e foram analisados de maneira descritiva. Resultados: Dos 72 pacientes avaliados, a maioria encontrava-se na faixa etária dos 50 aos 80 anos, 76,39\% eram tabagistas, com os principais sintomas descritos a tosse, dor torácica e dispneia, tendo a hemoptise como principal complicação do procedimento; dos 54 diagnósticos que foram conclusivos, 33\% foram carcinoma epidermóide e 20,38\% eram adenocarcinoma. Conclusão: Os pacientes submetidos à BPPTC eram na maioria de meia idade e idosos, tabagistas, referiam tosse, dor torácica e dispneia e possuíam o carcinoma epidermóide como diagnóstico final.

PALAVRAS-CHAVE: Biópsia por Punção. Biópsia Guiada por Imagem. Neoplasias Pulmonares. Carcinoma Epidermoide.

TCC 12 - PERFIL CLÍNICO EVOLUTIVO E TERAPÊUTICO DE PACIENTES COM MARCA-PASSO E CARDIODESFIBRILADOR IMPLANTÁVEL EM UM HOSPITAL UNIVERSITÁRIO

RESIDENTE: MARCONNY BATISTA LIMA

PROGRAMA: CLÍNICA MÉDICA 


\section{INSTITUIÇÃO: HOSPITAL UNIVERSITÁRIO DA UNIVERSIDADE FEDERAL DO PIAUÍ ORIENTADORES: CARLOS EDUARDO BATISTA DE LIMA}

INTRODUÇÃO: A estimulação cardíaca artificial surgiu com o objetivo de reduzir mortalidade associada aos bloqueios atrioventriculares. Hoje, são cerca de 700 mil novos marca-passos implantados por ano em todo o mundo melhorando a qualidade de vida e reduzindo a mortalidade associada às bradiarritmias. OBJETIVO: Avaliar o perfil clínico evolutivo e tratamento dos pacientes em uso de marca-passo ou cardiodesfibrilador implantável no Hospital Universitário da Universidade Federal do Piauí. METODOS: Tratase de um estudo transversal, do tipo observacional e descritivo no qual através da análise dos prontuários eletrônicos foram levantados os dados dos pacientes submetidos a procedimento envolvendo dispositivo cardíaco implantável de junho de 2015 a junho de 2018. RESULTADOS: Os procedimentos foram realizados em 189 pacientes apresentando um perfil com leve predomínio do sexo masculino (52,38\%), com faixa etária média de 70,37 anos (20-96 anos). O dispositivo mais implantado foi o marca-passo bicameral, com a principal indicação sendo o Bloqueio atrioventricular total $(47,62 \%)$. Tem-se um baixo número de complicações $(7,4 \%)$, sendo a principal complicação 0 deslocamento ou fratura de eletrodo. Os pacientes têm como principais comorbidades a hipertensão $(76,19 \%)$, insuficiência cardíaca congestiva $(20,63 \%)$ e diabetes $(14,81 \%)$. Quanto ao seguimento após realização de procedimento, 45,5\% dos pacientes fazem seguimento ambulatorial periodicamente. CONCLUSÃO: O procedimento mais realizado foi o implante de marca-passo bicameral com principal indicação sendo o BAVT. Encontrado um baixo número de complicações pós-procedimentos, menor do que as relatadas na literatura, e sempre solucionadas sem mais intercorrências. Os óbitos não tiveram associação direta com o implante de dispostivo cardíaco.

PALAVRAS-CHAVE: Bloqueio Atroventricular. Bradicardia. Marca-passo Artificial.

TCC 13 - LESÃO RENAL AGUDA EM PACIENTES SUBMETIDOS A CIRURGIA CARDÍACA

RESIDENTE: MARIA DARISA DE SOUSA POLICARPO

PROGRAMA: CLÍNICA MÉDICA

INSTITUIÇÃO: HOSPITAL UNIVERSITÁRIO DA UNIVERSIDADE FEDERAL DO PIAUÍ ORIENTADOR: RAIMUNDO ANTÔNIO CARDOSO JUNIOR

INTRODUÇÃO: A Lesão Renal Aguda (LRA) ocorre em até $30 \%$ dos pacientes submetidos à cirurgia cardíaca, sendo necessária diálise em aproximadamente $1 \%$ de todos os pacientes. Mesmo pequenas alterações na creatinina sérica estão relacionadas a um aumento da morbidade e mortalidade. OBJETIVO: Avaliar a frequência de LRA nos pacientes submetidos a Revascularização do Miocárdio e/ou Troca Valvar realizadas no Hospital Universitário da Universidade Federal do Piauí no ano de 2017. MÉTODOS: Estudo observacional e transversal, realizado por meio da coleta de dados de prontuários. As variáveis categóricas foram expressas como frequências e porcentagens e as variáveis contínuas como médias \pm desvios padrão. Os dados foram organizados em planilhas Excel e analisados com a utilização do aplicativo SPSS versão 20.0. RESULTADOS: Foram incluídos 98 pacientes. A média de idade foi de 58,6 anos, 52\% dos pacientes eram do sexo masculino e $87 \%$ pardos. Vinte e cinco pacientes $(25,5 \%)$ apresentaram LRA, sendo 84\% classificados como Estágio 1, 4\% Estágio 2 e 12\% Estágio 3. Dos pacientes do sexo masculino, 37,3\% apresentaram LRA, contrastando com $12,8 \%$ dos pacientes do sexo feminino. A média de idade dos pacientes que apresentaram LRA foi significativamente maior quando comparado aos que não tiveram disfunção. Dos 10 pacientes que evoluíram para o óbito, 7 apresentaram LRA no pós- 
operatório. CONCLUSÃO: Um percentual elevado de pacientes em pós-operatório de cirurgia cardíaca progrediu com Lesão renal aguda. Esses pacientes tiveram maior tempo de internação hospitalar e na UTI, além de evoluírem para óbito mais frequentemente. $O$ Estágio 1 da classificação AKIN para LRA foi o mais prevalente. Os níveis de creatinina sérica no pré-operatório estiveram relacionados a uma maior frequência de LRA no pósoperatório de cirurgia cardíaca, assim como as variáveis idade e sexo masculino.

PALAVRAS-CHAVE: Lesão Renal Aguda. Creatinina. Cirurgia Cardíaca.

TCC 14 - AVALIAÇÃO DO CONTROLE GLICÊMICO DOS PACIENTES DIABÉTICOS TIPO 2 EM USO DE ANTIDIABÉTICOS ORAIS E INSULINAS

RESIDENTE: PAULO HENRIQUE DE MELO FILHO

PROGRAMA: CLIINICA MÉDICA

INSTITUIÇÃO: HOSPITAL UNIVERSITÁRIO DA UNIVERSIDADE FEDERAL DO PIAUÍ ORIENTADOR: JOSÉ MARIA CORREIA LIMA E SILVA

INTRODUÇÃO: Diabetes Mellitus (DM) é um transtorno metabólico ocasionado pela alteração na produção ou na liberação da insulina pelas células beta. Segundo estimativa da Federação Internacional de Diabetes, cerca de 425 milhões de pessoas possuem DM. Vários estudos demonstraram que o controle glicêmico adequado previne complicações crônicas que constituem importantes causas de morbimortalidade. OBJETIVOS: Avaliar o controle glicêmico em diabéticos do tipo 2 acompanhados no ambulatório de endocrinologia do Hospital Universitário do Piauí em uso de antidiabéticos orais e insulinas dispensadas pela farmácia de medicamentos especializados. METODOLOGIA: Estudo observacional e transversal. As variáveis consideradas foram o nível de hemoglobina glicada (HbA1c), sexo, idade, procedência, tempo de diagnóstico, tempo de tratamento, frequência de consultas por ano, classe de medicamentos utilizado e presença de comorbidades. Os dados foram analisados no software $R$ versão 3.5.1. A amostra foi caracterizada por frequências absolutas e percentuais, média e desvio padrão, e utilizados teste de Anderson-Darling, Levene, $t$ de Student, $t$ de Student pareado, McNemar, qui-quadrado de Pearson e, por fim, foi calculada a razão de chances (odds ratio). RESULTADOS: Analisados 130 pacientes, 95 aptos a participar da pesquisa, $62,1 \%$ do sexo feminino. Frequência inicial de controle glicêmico adequado era de $15 \%$ e após tratamento passou para $48 \%$. Média inicial de HbA1c era de $8,6 \pm 1,60$ e após tratamento 7,4 $\pm 1,65$. CONCLUSÃO: Pacientes analisados obtiveram melhor controle comparando o início com o atual momento, evidenciado tanto pelo aumento do número de pacientes com $\mathrm{HbA} 1 \mathrm{c} \leq 7$, como pela redução da média de hemoglobina glicada.

PALAVRAS-CHAVE: Diabetes Mellitus. Controle Glicêmico. Hemoglobina Glicada.

TCC 15 - MAPEAMENTO ENDOSCÓPICO DE ÚLCERAS GÁSTRICAS

RESIDENTE: PRISCILA DA SILVEIRA SANTOS

PROGRAMA: ENDOSCOPIA DIGESTIVA

INSTITUIÇÃO: HOSPITAL UNIVERSITÁRIO DA UNIVERSIDADE FEDERAL DO PIAUÍ ORIENTADOR: JEANY BORGES E SILVA RIBEIRO

INTRODUÇÃO: A úlcera péptica clássica ocorre por quebra na barreira mucosa do estômago ou duodeno, através da muscular da mucosa, com alta morbimortalidade. Frequência e riscos variam segundo aspectos clínicos, sociodemográficos e geográficos. A endoscopia digestiva alta (EDA) é o exame padrão-ouro para detecção. As complicações incluem sangramento, perfuração, obstrução e malignidade. Biópsias de 
úlceras gástricas (UG) são frequentemente necessárias para detecção de neoplasias precoces. OBJETIVOS: Realizar o mapeamento de UG, segundo características endoscópicas, histopatológicas, variáveis demográficas, associação com Helicobacter pylori, com úlceras duodenais (UD) e risco de gravidade. METODOLOGIA: Estudo seccional, descritivo-analítico e retrospectivo, com dados de 01/01/2016 a 05/07/2018 coletados em prontuários médicos. RESULTADOS: Foram avaliados 5137 laudos endoscópicos, com 207 indivíduos apresentando UG (4,02\%) e um total de 265 UG detectadas. Dos acometidos, 121 eram mulheres $(58,5 \%)$ e a idade média foi de 59,69 anos. $80,4 \%$ das lesões localizavam-se em antro, sendo o sítio mais observado em ambos os sexos. Houve associação com UD em 14 casos. Observou-se maior frequência de úlceras A1 de Sakita e Forrest III. Do total, 116 foram positivas para Helicobacter pylori e a maioria benigna $(81,8 \%)$. Sexo e idade não interferiram nas classificações de Sakita, Forrest e nem na localização das lesões. CONCLUSÃO: Houve 207 indivíduos afetados por UG e um total de 265 lesões, a maioria delas ativa, benigna, sem sangramento, associadas com Helicobacter pylori nas biopsiadas. A maior frequência desta patologia ocorreu no antro e no sexo feminino. Sexo e idade não interferiram nas medidas de risco das UG.

PALAVRAS-CHAVE: Úlcera Gástrica. Úlcera Duodenal. Endoscopia Gastrointestinal. Helicobacter Pylori. Neoplasias Gástricas.

TCC 16 - AVALIAÇÃO DOS NÍVEIS DE VITAMINA B12 EM PACIENTES DIABÉTICOS COM USO DE METFORMINA

RESIDENTE: ISADORA NOLETO BARBOSA

PROGRAMA: ENDOCRINOLOGIA

ORIENTADOR: ANDRÉ GONÇALVES DA SILVA

INTRODUÇÃO: Metformina é a medicação inicial preferencial para Diabetes mellitus tipo 2 e pode estar associada a deficiência de vitamina B12. OBJETIVO: Avaliar os níveis de vitamina B12 e fatores associados em pacientes diabéticos, com uso de metformina há pelo menos 6 meses, analisando-se, ainda, se a chance de ter baixo nível da vitamina é maior nos pacientes que usam metformina do que nos que não usam e analisando-se a presença de sintomas de neuropatia periférica entre os diabéticos submetidos a dosagem de vitamina B12. METODOLOGIA: Estudo transversal, observacional. Incluíram-se diabéticos em uso de metformina e um grupo controle de diabéticos sem metformina. Investigou-se a associação entre vitamina B12 (normal ou deficiente) e idade, sexo, dose de metformina, uso de inibidor de bomba de prótons (IBP) e antagonistas de $\mathrm{H} 2$ nos usuários da biguanida, e pesquisaram-se sintomas de neuropatia periférica entre os participantes. RESULTADOS: Houve 135 participantes, 11,71\% com deficiência de vitamina B12 e 12,59\% com sintomas de neuropatia periférica. Não houve associação entre idade, sexo, dose de metformina, uso de IBP e antagonistas de H2 com a deficiência vitamínica. Não houve significância no teste para associação entre a medicação e a hipovitaminose. CONCLUSÃO: A frequência de deficiência de vitamina B12 foi menor do que em outros serviços de saúde e não houve indícios de associação com as variáveis pesquisadas. Parte considerável dos pacientes relataram sintomas de neuropatia periférica. Não foi possível afirmar se a chance de ter baixo nível de vitamina B12 é maior nos pacientes que usam metformina.

PALAVRAS-CHAVE: Vitamina B12. Metformina. Diabetes Mellitus. 
TCC 17 - EFEITOS DA MEDITAÇÃO GUIADA SOBRE O BEM-ESTAR FÍSICO E EMOCIONAL DE PACIENTES IDOSOS EM TRATAMENTO ONCOLÓGICO

RESIDENTE: LAIZA CAVALCANTE DE CARVALHO CUNHA

PROGRAMA: GERIATRIA

INSTITUIÇÃO: HOSPITAL UNIVERSITÁRIO DA UNIVERSIDADE FEDERAL DO PIAUÍ

ORIENTADOR: GLENDA MARIA SANTOS MOREIRA

INTRODUÇÃO: A meditação faz parte de um grupo de terapias denominado de Terapias Integrativas, que compreendem um grupo de práticas de atenção à saúde não alopáticas. A Organização Mundial da Saúde vem estimulando o uso destas terapias de forma racional e segura. OBJETIVOS: O propósito do presente estudo foi avaliar o efeito da meditação sobre o bem estar físico e emocional de pacientes idosos em tratamento oncológico no ambulatório do HU-UFPI. MÉTODOS: O delineamento experimental foi de um ensaio clínico randomizado. Todos os pacientes em tratamento no serviço de oncologia do HU-UFPI foram convidados a participar voluntariamente do estudo. Os voluntários que preencheram os critérios de elegibilidade foram alocados aleatoriamente em um dos dois grupos, Meditação ou Controle. Os indivíduos do grupo Meditação foram instruídos a praticar meditação guiada por áudio durante 15 minutos, uma vez ao dia, durante 03. Todos os voluntários responderam ao questionário para avaliação de qualidade de vida no primeiro e último dia do estudo. O bem estar físico e emocional dos participantes foi avaliado pelo questionário EORTC-QLQ-C30. Os dados obtidos foram analisados por meio de testes estatísticos adequados. RESULTADOS: Observa-se que houve diferença estatisticamente significativa entre as medianas das diferenças dos grupos meditação e controle no domínio do estado de saúde global e no domínio funcional. Já no domínio dos sintomas essa diferença não foi detectada. CONCLUSÃO. A meditação guiada mostrou-se capaz de melhorar aspectos relacionados ao bem estar dos praticantes, após um curto período de 21 dias de meditação.

PALAVRAS-CHAVE: Meditação. Qualidade de Vida. Saúde Mental

TCC 18 - FREQUÊNCIA DE SÍNDROME DO TÚNEL DO CARPO ASSOCIADA A DEDO EM GATILHO EM PACIENTES ATENDIDOS EM HOSPITAL UNIVERSITARIO DO PIAUÍ RESIDENTE: LEONARDO ANTONIO DE CARVALHO HIPÓLITO

PROGRAMA: ORTOPEDIA E TRAUMATOLOGIA

INSTITUIÇÃO: HOSPITAL UNIVERSITÁRIO DA UNIVERSIDADE FEDERAL DO PIAUÍ ORIENTADOR: OSVALDO MENDES DE OLIVEIRA FILHO

INTRODUÇÃO: A síndrome do túnel do carpo é a mais comum neuropatia periférica compressiva, ocorre em $0,1 \%$ a $10 \%$ da população e é diagnosticada com precisão na maioria dos casos. OBJETIVO: Determinar frequência de túnel do carpo associada a dedo em gatilho. MÉTODOS: A pesquisa foi um estudo transversal, observacional, de caráter exploratório descritivo, visto que os fatos foram observados, registrados, analisados, classificados e interpretados, sem a interferência do pesquisador, através de questionário, o qual buscou apenas levantar informações sobre um determinado objeto, delimitando assim um campo de trabalho e mapeando as condições de manifestação desse objeto. RESULTADOS: Foram pesquisados 31 pacientes com síndrome do túnel do carpo. Desses, 31 pacientes (100\%) são do sexo feminino, com idade entre 40 e 60 anos. Quando perguntados sobre o tempo de início dos sintomas, mais especificamente a parestesia, todos os 31 pacientes $(100 \%)$ relataram ter o sintoma há mais de um ano. Quanto aos testes especiais realizados na pesquisa para síndrome do túnel do carpo, todos os 31 pacientes (100\%) apresentaram sinal de Phalen e Dunkan positivo ao exame 
físico. 29(70,9\%) apresentavam sinal Tinel e Paley positivos. Cinco pacientes (58\%) apresentavam Phalen positivo em menos de 30 segundos. CONCLUSÃO: A pesquisa mostrou uma alta frequência de síndrome do túnel do carpo com sinais clínicos e eletroneuromiograficos de gravidade com dedo em gatilho, corroborando com outros estudos.

PALAVRAS-CHAVE: Parestesia. Nervomediano. Tenossinovite.

TCC 19 - TESTE DE PHALEN MODIFICADO NA SÍNDROME DO TÚNEL DO CARPO

RESIDENTE: THÚLIO ADLEY LIMA CUNHA

PROGRAMA: ORTOPEDIA E TRAUMATOLOGIA

INSTITUIÇÃO: HOSPITAL UNIVERSITÁRIO DA UNIVERSIDADE FEDERAL DO PIAUÍ

ORIENTADOR: OSVALDO MENDES DE OLIVEIRA FILHO

INTRODUÇÃO: A síndrome do túnel do carpo (STC) foi inicialmente descrita por Paget em 1984. É considerada a neuropatia mais comum nos membros superiores. E caracteriza-se pela compressão do nervo mediano ao nível do túnel do carpo, no punho, devido ao aumento do tamanho das estruturas contidas em seu interior, ou em razão da diminuição de suas dimensões. A Eletroneuromiografia (ENMG) é o principal exame para o diagnóstico desta neuropatia. OBJETIVO: Comparar a classificação pelo teste de Phalen modificado com a eletroneuromiografia na STC. MÉTODOS: Estudo descritivo transversal; Pacientes com síndrome do túnel do carpo do ambulatório de ortopedia do HUUFPI avaliados pelo teste de Phalen modificado e comparado ao resultado da eletroneuromiografia. RESULTADOS: Amostra de 33 pacientes, em sua maioria mulheres $(87,9 \%)$, Diabetes Mellitus como comorbidade mais frequente $(71,4 \%)$. Maioria dos pacientes com STC grave pela ENMG. Os resultados do teste de Phalen modificado e da eletroneuromiografia foram iguais em 26 dentre os 33 pacientes avaliados $(78,8 \%)$. CONCLUSÃO: O teste de Phalen modificado mostrou ter sua aplicabilidade, pois teve resultados semelhantes aos da ENMG na maioria dos casos, principalmente nos mais graves. O exame em estudo se mostra uma possível ferramenta de classificação e indicação do tratamento cirúrgico.

PALAVRAS-CHAVE: Síndrome do Túnel Carpal. Nervo Mediano. Punho.

TCC 20 - CAPACIDADE PREDITIVA DO ESCORE DE WELLS NO DIAGNÓSTICO DE TROMBOSE VENOSA PROFUNDA

RESIDENTE: LUIZA ÊNIA DA SILVA ARAÚJO

PROGRAMA: RADIOLOGIA E DIAGNÓSTICO POR IMAGEM

INSTITUIÇÃO:

ORIENTADOR: EDUARDO BRUNO LOBATO MARTINS

INTRODUÇÃO: O diagnóstico precoce de trombose venosa profunda é importante para o tratamento eficaz e prevenção de consequências mais graves, como insuficiência venosa crônica, síndrome pós-trombótica e a embolia pulmonar que pode ser letal. OBJETIVO: Avaliar a capacidade preditiva do escore de Wells para pacientes com suspeita de trombose venosa profunda. METODOLOGIA: Delineamento transversal analítico, com avaliação preditiva de um modelo para trombose venosa profunda, realizado em um hospital universitário. A amostra ficou composta por 24 pacientes. Foi utilizado um formulário para obtenção de informações sociodemográficas e epidemiológicas, bem como o Escore de Predição de Wells. Foram calculadas estatísticas uni e bivariadas. O estudo foi aprovado por Comitê de Ética. RESULTADOS: A média de idade dos pacientes 
foi $53,3( \pm 16,8)$ anos, variando de 22,0 a 84,0 anos, com maioria do sexo feminino $(62,5 \%)$. A média dos escores de predição na amostra deste estudo foi de $1,2( \pm 2,4)$ pontos, com mínimo $-2,0$ e máximo 5,0 . Os pacientes, distribuídos igualmente quanto ao risco provável $(50,0 \%)$ e improvável $(50,0 \%)$. Os valores de sensibilidade, especificidade, valor preditivo positivo e negativo, razão de verossimilhança negativa e positiva, precisão, eficiência e coeficiente de correlação de Mathews foram: 100,0\%, 60,0\%, 33,3\%, 100,0\%, 2,5, 0,0, 66,7\%, 80,0\% e 0,447. CONCLUSÃO: O Escore de Predição de Wells apresentou-se como recurso complementar confiável para excluir trombose venosa profunda nos pacientes do hospital universitário.

PALAVRAS-CHAVE: Trombose Venosa Profunda. Escore de Wells. Ultrassonografia Doppler.

TCC 21 - NEFROLITÍASE: COMPARAÇÃO ENTRE ULTRASSONOGRAFIA E TOMOGRAFIA COMPUTADORIZADA

RESIDENTE: RENAN BASTOS LEITE

PROGRAMA: RADIOLOGIA E DIAGNÓSTICO POR IMAGEM

INSTITUIÇÃO: HOSPITAL UNIVERSITÁRIO DA UNIVERSIDADE FEDERAL DO PIAUII ORIENTADOR: AILA DE MENEZES FERREIRA

INTRODUÇÃO: A nefrolitíase é uma condição altamente prevalente na população. Dentre os exames disponíveis para diagnóstico, a Tomografia Computadorizada (TC) destaca-se pela elevada sensibilidade e possibilidade de realizar diagnósticos diferenciais. A ultrassonografia (US), apesar da baixa sensibilidade, continua sendo utilizada pela boa disponibilidade e não utilização de radiação ionizante. OBJETIVOS: Comparar a acurácia da US em relação à TC na identificação de nefrolitíase nos pacientes que realizaram os dois exames, verificando erro no dimensionamento dos cálculos através da US tendo como base a TC, identificando o tamanho mínimo onde ambos se equivalem. METODOLOGIA: Estudo observacional, transversal, descritivo, com coleta retrospectiva dos dados de pacientes com diagnóstico de nefrolitíase no Hospital Universitário da Universidade Federal do Piauí (HU-UFPI) através de TC das vias urinárias e que realizaram exame de US no mesmo serviço, em um intervalo inferior a 06 meses. RESULTADOS: Foram avaliados 111 pacientes com nefrolitíase. Do total, 54,1\% eram do sexo feminino e $45,9 \%$ do sexo masculino. A totalidade dos pacientes possuía achado tomográfico de nefrolítiase e 47,7\% possuía achado ultrassonográfico concordante. A taxa de detecção do US comparado à TC foi de $36,2 \%$ à direita e $42,1 \%$ à esquerda. Observou-se alta correlação entre US e TC para cálculos com dimensões superiores a 0,7 $\mathrm{cm}$, baixa correlação entre $0,31 \mathrm{~cm}$ e $0,7 \mathrm{~cm}$ e ausência de correlação abaixo de $0,3 \mathrm{~cm}$. CONCLUSÃO: Quanto maiores as dimensões dos cálculos renais maior a correlação dos achados entre US e TC. A US é eficiente para cálculos maiores do que 0,7 cm.

PALAVRAS-CHAVE: Nefrolitíase. Tomografia Computadorizada. Ultrassonografia.

TCC 22 - ABORDAGEM DA SEXUALIDADE EM CONSULTAS AMBULATORIAIS POR GINECOLOGISTAS E OBSTETRAS

RESIDENTE: ALESSANDRA CARVALHO DE OLIVEIRA

PROGRAMA: OBSTETRÍCIA E GINECOLOGIA

INSTITUIÇÃO:

ORIENTADOR: ANAÍDE ROSA DE CARVALHO NASCIMENTO PINHEIRO 
INTRODUÇÃO: O atendimento médico integral deve incorporar tópicos de saúde sexual, que podem ser negligenciados ou evitados. OBJETIVO: Avaliar a abordagem da sexualidade em consultas ambulatoriais realizadas por ginecologistas e obstetras. METODOLOGIA: Delineamento transversal analítico, realizado com uma amostra de conveniência composta por 57 ginecologistas e/ou obstetras registrados no Conselho de Medicina estadual. Foram coletadas variáveis sociodemográficas, educacionais e relacionadas ao atendimento. A avaliação da abordagem da sexualidade foi realizada por meio de um instrumento elaborado pelas autoras, composto por cinco itens e duas seções. Foram realizadas análises uni e bivariadas com IBM® SPSS $\circledast$ 23.0. O estudo foi aprovado por Comitê de Ética. RESULTADOS: A média do nível de abordagem da sexualidade quanto à iniciativa e segurança do médico ginecologista e obstetra foi de $60,5 \%$, enquanto para os aspectos práticos referidos pelas pacientes foi de $70,2 \%$. 0 escore global foi de, em média, $65,4 \%$, variando de $16,7 \%$ a $91,7 \%$, sendo que $14,0 \%$ dos médicos atingiram nível $0 \%$ nas autoavaliações. Nas medidas do domínio iniciativa e segurança e do escore global, verificou-se que a abordagem da sexualidade foi significativamente inferior nos profissionais que atuam somente em obstetrícia $(p=0,010$ e $p=0,014)$. Maiores níveis de abordagem foram verificados para os profissionais que relataram desenvolver atividades acadêmicas $(p=0,010$ e $p=0,007)$. CONCLUSÂO: Observou-se que o maior nível de abordagem referiu-se aos aspectos práticos, demonstrando uma iniciativa maior por parte das pacientes para discussão do tema do que pelos profissionais.

PALAVRAS-CHAVE: Ginecologia. Obstetrícia. Sexualidade. Saúde Sexual.

TCC 23 - SATISFAÇÃO DAS PUÉRPERAS PÓS-PARTO VAGINAL EM MATERNIDADE DE REFERÊNCIA DO PIAUÍ

RESIDENTE: CARLOS EDUARDO ALMEIDA SOUSA

PROGRAMA: OBSTETRÍCIA E GINECOLOGIA

INSTITUIÇÃO: MATERNIDADE DONA EVANGELINA ROSA

ORIENTADOR: ANA MARIA COÊLHO HOLANDA

INTRODUÇÃO: O período da gestação e parto constitui um dos momentos mais importantes da vida da mulher, com o envolvimento do parceiro e sua família. A assistência humanizada representa um novo paradigma que proporciona à mulher benefícios físicos e psicológicos, permitindo-a ocupar o cerne de todo o processo. A avaliação da assistência ao parto faz-se necessária para verificar a percepção das pacientes frente ao novo modelo implantado. OBJETIVO: Verificar a satisfação com o parto vaginal em puérperas atendidas na Maternidade Dona Evangelina Rosa. METODOLOGIA: Estudo transversal e descritivo, realizado na Maternidade Dona Evangelina Rosa. Utilizou-se como instrumento de coleta a escala de Bem-estar Materno em Situação de Parto tipo 2, produzida no Chile e traduzida e adaptada para o português. RESULTADOS: Participaram deste estudo 380 puérperas, sendo 176 primíparas, 170 multíparas e $34 \mathrm{com}$ histórico de cesariana anterior. O perfil mais prevalente foi mulher jovem, com média de idade de 25,8 anos $( \pm 6,3)$, cor parda, com ensino médio completo, em união estável, sem exercício de atividade remunerada e de baixa renda. A pontuação na escala foi ótima (55\%), adequada (26\%) e mal-estar (19\%). Não houve diferença estatisticamente significativa entre o bem-estar materno e as variáveis analisadas (planejamento da gravidez, realização de episiotomia, paridade, tempo de permanência no pré-parto e participação em palestras educativas no pré-natal). CONCLUSÃO: As pacientes submetidas ao parto vaginal apresentaram elevado grau de bem-estar e este dado pode ser utilizado para estimular a escolha desta via de parto e reduzir o número de cesarianas. 
PALAVRAS-CHAVE: Parto Normal. Bem-Estar Materno. Satisfação do Usuário.

TCC 24 - ATIVIDADE SEXUAL E QUALIDADE DE VIDA DE MULHERES SUBMETIDAS A HISTERECTOMIA PARA TRATAMENTO DE LEIOMIOMA UTERINO

RESIDENTE: FELIPE OSÓRIO MARQUES

PROGRAMA: OBSTETRÍCIA E GINECOLOGIA

INSTITUIÇÃO:

ORIENTADOR: ANAÍDE ROSA DE CARVALHO NASCIMENTO PINHEIRO

INTRODUÇÃO: A histerectomia resulta em melhoria dos sintomas do leiomioma uterino, porém, especula-se que a extirpação desse órgão possa prejudicar a função sexual. OBJETIVO: Avaliar a atividade sexual e a qualidade de vida de mulheres submetidas a histerectomia para tratamento de leiomioma uterino. METODOLOGIA: Delineamento transversal analítico, realizado com pacientes que foram submetidas a histerectomia abdominal devido ao diagnóstico de leiomioma uterino. A amostra de conveniência totalizou 36 participantes. Foram coletadas variáveis socioeconômicas, tocoginecológicas e clínicas. Foi utilizado o Quociente Sexual - Versão Feminina (QS-F) para medida do padrão de desempenho sexual e a versão brasileira do World Health Organization Quality of Life instrument short 26-question version (WHOQOL-bref) para qualidade de vida. Foram realizadas análises uni e bivariadas com IBM ${ }^{\circledR}$ SPSS ${ }^{8}$ 23.0. O estudo foi aprovado por Comitê de Ética. RESULTADOS: Foi verificada correlação estatisticamente significativa entre o desempenho sexual global e a qualidade de vida global das mulheres pós-histerectomia por leiomioma uterino $(p=0,020)$, destacando-se correlações pontuais com a percepção subjetiva da qualidade de vida $(p=0,012)$ e a satisfação com a saúde $(p=0,006)$, além do domínio psicológico $(p=0,026)$. O domínio de desempenho sexual que mais apresentou correlações com a qualidade de vida foi orgasmo e satisfação sexual. CONCLUSÃO: O nível global de satisfação sexual das pacientes histerectomizadas devido leiomioma uterino apresentou-se regular, com quase $40 \%$ apresentando desempenho bom a excelente e a qualidade de vida geral regular. As correlações encontradas apontaram relações entre o desempenho sexual e o nível de qualidade de vida dessas mulheres.

PALAVRAS-CHAVE: Leiomioma uterino. Histerectomia. Qualidade de Vida. Saúde Sexual.

TCC 25 - FREQUÊNCIA E FATORES DE RISCO RELACIONADOS ÀS LESÕES PERINEAIS GRAVES EM PARTURIENTES

RESIDENTE: LORENA NORBERTA MENDES MOURA

PROGRAMA: OBSTETRÍCIA E GINECOLOGIA

INSTITUIÇÃO: MATERNIDADE DONA EVANGELINA ROSA

ORIENTADOR: ANA MARIA COÉLHO HOLANDA

INTRODUÇÃO: As lacerações pélvicas de natureza obstétrica são classificadas, conforme a extensão dos tecidos acometidos, em leves e graves. Essas são fatores de risco independentes, para os agravos a saúde perineal que impactam negativamente na qualidade de vida da mulher. A importância da prevenção primária motiva a determinação das variáveis materno / fetais / assistenciais implicados em maior frequência de injúrias pélvicas graves. OBJETIVO: Identificar os fatores associados às lacerações perineais graves em pacientes submetidas a parto vaginal na Maternidade Dona Evangelina Rosa em Teresina - PI. METODOLOGIA: Trata-se de um estudo observacional, analítico e transversal. A amostra foi composta por mulheres que tiveram parto 
Maternidade Dona Evangelina Rosa respeitados os critérios de inclusão e exclusão. A coleta ocorreu por meio questionários estruturados. Os dados coletados foram submetidos à análise, através das ferramentas estatísticas de frequência, testes quiquadrado e teste Exato de Fisher. RESULTADOS: Participaram do estudo 164 parturientes, a média de idade foi de 25 anos, ocorreram lacerações graves em 3,04\% dos casos, $91,25 \%$ do total de injúrias pélvicas foram submetidos à correção cirúrgica. Quanto ás variáveis investigadas para laceração obstétrica grave observou-se que essa ocorreu em $6,4 \%$ das primíparas, $14,3 \%$ das mulheres que receberam analgesia farmacológica, $9,3 \%$ das que tiveram parto induzido, $25 \%$ das que tiveram extração fetal por fórceps, $16,8 \%$ das mulheres submetidas à episiotomia. Variedades de posição fetal anterior e posterior foram relacionadas a menor número de traumas graves. CONCLUSÃO: A frequência de lacerações perineais graves pode ser considerada baixa, fatores de risco associados são, primiparidade, uso de analgesia farmacológica, indução de trabalho de parto, uso de fórceps e episiotomia. A posição fetal em occipito anterior e posterior foram fatores de proteção. A conduta obstétrica deve ser orientada, de modo a aumentar a probabilidade de desfechos perineais favoráveis.

PALAVRAS - CHAVES: Laceração Perineal. Parto Vaginal. Fatores de Risco.

TCC 26 - FREQUÊNCIA DE FATORES AMBIENTAIS E COMPORTAMENTAIS ASSOCIADOS AO CÂNCER DE COLO UTERINO EM MULHERES ATENDIDAS EM HOSPITAL UNIVERSITÁRIO DO PIAUÍ

\section{RESIDENTE: MARIA CLARA FORTES PORTELA BONFIM \\ PROGRAMA: OBSTETRÍCIA E GINECOLOGIA \\ INSTITUIÇÃO: \\ ORIENTADOR: MARTA ALVES ROSAL}

INTRODUÇÃO: O câncer de colo uterino é a $2^{\mathrm{a}}$ causa de morte entre todos os tipos de cânceres da mulher. Os fatores de risco associados à oncogênese cervical incluem fatores imunológicos, fatores genéticos, fatores ambientais e comportamentais como: tabagismo, uso de contraceptivos, início precoce da vida sexual precoce, múltiplos parceiros, baixa escolaridade e renda, multiparidade e história de doenças sexualmente transmissíveis. OBJETIVO: Caracterizar os fatores de risco ambientais e comportamentais presentes em pacientes com neoplasia intraepitelial cervical (NIC) e câncer de colo uterino. MÉTODOS: Estudo transversal, descritivo, com abordagem quantitativa, desenvolvido em grupo de 71 mulheres com diagnóstico de NIC e câncer de colo uterino atendidas no período de março de 2018 a dezembro de 2018. Foram coletados dados mediante busca no prontuário eletrônico e aplicação de questionários às pacientes. RESULTADOS: No grupo de mulheres estudadas, a maioria possuía renda mensal inferior e três salários mínimos (93\%) e tinha ensino fundamental incompleto (69\%); quanto aos fatores ambientais e comportamentais de risco para o câncer de colo uterino, $56,3 \%$ eram multíparas, 33,8\% eram tabagistas ou ex-tabagistas e $29,6 \%$ fizeram uso de anticoncepcional por no mínimo 05 anos. O início da vida sexual ocorreu em torno de 17,5 anos, média de 3,4 parceiros e 5,2 exames de citologia oncótica realizados em 10 anos. CONCLUSÃO: Os fatores ambientais e comportamentais associados ao câncer de colo uterino mais presentes no grupo estudado foram: baixa renda, baixa escolaridade, múltiplos parceiros e início precoce da vida sexual.

PALAVRAS-CHAVE: Neoplasia do Colo do Útero. Neoplasia Intraepitelial Cervical. Fatores de Risco. 
TCC 27 - FATORES PREDITORES DE INCONTINÊNCIA URINÁRIA CONFIRMADA EM ESTUDO URODINÂMICO EM MULHERES

RESIDENTE: THAINÁ PINTO DOS SANTOS

PROGRAMA: OBSTETRÍCIA E GINECOLOGIA

INSTITUIÇÃO: HOSPITAL UNIVERSITÁRIO DA UNIVERSIDADE FEDERAL DO PIAUÍ

ORIENTADOR: PEDRO VITOR LOPES COSTA

INTRODUÇÃO: Importantes repercussões psicossociais e econômicas podem afetar a qualidade de vida de mulheres com incontinência urinária. OBJETIVO: Analisar os fatores preditores de incontinência urinária confirmada por meio de estudo urodinâmico em mulheres. METODOLOGIA: Delineamento transversal controlado, realizado com mulheres em atendimento ambulatorial encaminhadas ao setor de Saúde da Mulher de um hospital universitário. A amostra do estudo foi composta por 68 mulheres com incontinência urinária confirmada por meio de estudo urodinâmico e 68 mulheres sem incontinência urinária, totalizando 136 participantes. A amostragem foi não probabilística, do tipo por conveniência. Foram coletadas variáveis sociodemográficas, epidemiológicas e obstétricas. Foi utilizado o modelo de regressão logística com estimador robusto por meio do IBM® SPSS $\AA^{2}$ 23.0. RESULTADOS: Seis variáveis foram identificadas como fatores preditores de incontinência urinária confirmada por estudo urodinâmico em mulheres: uso de diuréticos $\mathrm{RP}=10,149$ (IC95\%=2,692-38,261), constipação $\mathrm{RP}=7,169$ (IC95\%=2,28022,538), número de gestações $\mathrm{RP}=2,263$ (IC95\%=1,019-5,024), número de partos $\mathrm{RP}=2,826$ (IC95\%=1,089-7,338), maior número de partos com episiotomia $\mathrm{RP}=2,599$ (IC95\%=1,386-4,873) e peso as nascer $\mathrm{RP}=7,334$ (IC95\%=1,505-35,743). CONCLUSÃO: Fatores sociodemográficos, epidemiológicos e obstétricos foram associados à presença de incontinência urinária em mulheres, confirmada por meio do estudo urodinâmico, sendo que seus preditores apresentaram-se especialmente nos dois últimos agrupamentos. Conhecer os fatores preditores pode contribuir para o desenvolvimento de projetos educativos e capacitação dos profissionais da saúde, a fim de que sejam realizados trabalhos para conscientização da população feminina quanto aos fatores de risco modificáveis.

PALAVRAS-CHAVE: Saúde da Mulher. Incontinência Urinária. Fatores de Risco. Urodinâmica.

TCC 28 - CONHECIMENTO DE CUIDADORES DE CRIANÇAS E ADOLESCENTES SOBRE SEGURANÇA NO TRÂNSITO

\section{RESIDENTE: ANDRESSA DANIELA DE SOUSA PROGRAMA: PEDIATRIA INSTITUIÇÃO: \\ ORIENTADOR: SIMONE SOARES LIMA}

INTRODUÇÃO: Os acidentes de trânsito na população pediátrica podem levar à incapacidade permanente e óbito, ocorrem por desconhecimento e desobediência às normas de segurança. OBJETIVO: Avaliar o conhecimento de cuidadores de crianças e adolescentes sobre segurança no trânsito e os fatores relacionados. Identificar adesão, caracterizar acidentes e o meio de informação. MÉTODOS: Estudo transversal, observacional e quantitativo. Entrevistou-se cuidadores de crianças e adolescentes em atendimento ambulatorial sobre o conhecimento e adesão às normas de trânsito; e perfil socioeconômico-cultural e demográfico do cuidador. Utilizou-se escore de porcentagens de acertos para análise do conhecimento, testes bicaudais e um nível de significância a 
de 0,05. RESULTADOS: Entrevistou-se 165 cuidadores, principalmente mães (89,7\%), procedentes do interior do Piauí $(53,3 \%)$, renda familiar menor ou igual a um salário mínimo $(79,4)$, ensino médio completo $(43 \%)$ e até dois menores sob supervisão $(72,1 \%)$. A maioria não recebeu orientação para o trânsito $(72,7 \%)$, a principal fonte foi a escola $(62,2 \%)$. A família usava motocicleta como principal transporte $(52,7 \%)$, negavam acidente infantil $(94,5 \%)$. O grau de adesão $(35,86 \% \pm 31,48)$ e conhecimento $(41,82 \pm$ $20,72)$ foram baixos e relacionados entre si $(r=0,169 ; p=0,011)$. Este relacionado à procedência de outros Estados $(p<0,01)$, ensino superior completo $(p<0,01)$, renda superior a um salário $(p=0,022)$ e até dois menores sob supervisão $(p=0,034)$. CONCLUSÃO: O grau de conhecimento e adesão às normas de trânsito por cuidadores são baixos e relacionados entre si. Ocorre maior conhecimento quando há maior escolaridade e renda e menor número de crianças supervisionadas.

Palavras-chave: Acidentes de Trânsito. Crianças. Adolescentes. Conhecimento. Cuidadores.

TCC 29 - RELAÇÃO ENTRE A TRANSMISSÃO VERTICAL DO HIV E AS CONDIÇÕES DA GRAVIDEZ E DO PARTO

RESIDENTE: BEATRIZ DE OLIVEIRA FREIRE

PROGRAMA: PEDIATRIA

INSTITUIÇÃO:

ORIENTADOR: DORCAS LAMOUNIER

INTRODUÇÃO: A transmissão do Vírus da Imunodeficiência Humana (HIV) de mães para crianças pode acontecer durante a gestação e pela amamentação. Apesar de medidas de profilaxia serem bem conhecidas, a transmissão vertical ainda é principal via de infecção em crianças no Brasil. A identificação dos fatores de risco é essencial para a compreensão da epidemiologia e para a implementação de políticas públicas para a eliminação da transmissão vertical do HIV. OBJETIVOS: Avaliar as condições da gestação e do parto e o aleitamento materno como fatores de risco para aquisição da infecção em crianças expostas verticalmente ao HIV, em um serviço de referência em infectologia pediátrica. METODOLOGIA: Estudo caso-controle retrospectivo. A população de estudo compreendeu filhos de mulheres infectadas pelo HIV, que nasceram de janeiro de 2006 a dezembro de 2017 atendidos no ambulatório de infectologia pediátrica. A análise estatística foi realizada pelo programa Stata/SE® 10.0. RESULTADOS: Foram identificadas 286 crianças expostas ao HIV e 276 preencheram os critérios de inclusão. Foram randomizadas 40 crianças infectadas e 120 crianças não infectadas. A infecção da criança esteve associada ao número de consultas realizadas durante o pré-natal, ao aleitamento materno, à terapia antirretroviral durante a gravidez, durante o trabalho de parto e no período neonatal, à via de parto e à escolaridade materna. CONCLUSÃO: A infecção de crianças pelo HIV é evitável na maior parte das vezes. A melhoria da educação de meninas e o fortalecimento das ações de atenção básica à saúde se apresentam como as principais medidas a serem implementadas.

PALAVRAS-CHAVE: Crianças. HIV. Transmissão Vertical de Doença Infecciosa. Fatores de Risco.

TCC 30 - CONHECIMENTO MATERNO SOBRE O TESTE DO PEZINHO EM MATERNIDADE DE REFERÊNCIA DO PIAUÍ

RESIDENTE: DANIELE DE OLIVEIRA RODRIGUES

PROGRAMA: PEDIATRIA 


\section{INSTITUIÇÃO: \\ ORIENTADOR: DÁYRTON RAULINO MOREIRA}

INTRODUÇÃO: A triagem neonatal permite rastrear indivíduos com determinadas patologias, realizando o diagnóstico em estágio precoce e instituindo tratamento específico, de modo a evitar sequelas graves e aumentar a sobrevida dos acometidos. OBJETIVO: Investigar o conhecimento das mães quanto à realização do teste do pezinho em maternidade de referência do Estado do Piauí. METODOLOGIA: Estudo transversal e quantitativo. Foram entrevistados 375 puérperas através de formulário com questões referentes ao perfil sócio-demográfico das mesmas além de perguntas relacionadas ao conhecimento materno sobre teste do pezinho. RESULTADOS: Verificou-se que o perfil das entrevistadas, 34,5\% tinham idade entre 18 e 23 anos, casadas ou união estável $(65,2 \%)$, ensino médio completo $(40,8 \%)$, renda mensal de um salário mínimo $(46,7 \%)$, possui apenas um filho (45,1\%), realizaram seis ou mais consultas pré-natal $(70,9 \%)$ e procedência urbana $(60,9 \%)$. Quase todas já ouviram falar sobre o teste do pezinho $(99,5 \%)$ e $53 \%$ receberam as informações durante o pré natal. Todas as puérperas acharam o teste importante e 51,4\% sabiam a finalidade do teste, entretanto, 68,2\% não sabiam o período ideal para realizar o teste e $97,8 \%$ não sabiam citar as doenças triadas. Verifica-se que $48,2 \%$ das puérperas afirmaram que foi a enfermeira quem orientou sobre teste do pezinho. CONCLUSÃO: O conhecimento das puérperas sobre o teste do pezinho é superficial e pode ser reflexo da fragilidade de atuação da equipe de saúde. É necessário priorizar ações de educação nos serviços de saúde, visando melhorar a qualidade da assistência prestada à mãe e ao recém-nascido.

PALAVRAS-CHAVE: Pediatria. Teste do Pezinho. Conhecimento. Mães.

TCC 31 - CONHECIMENTO DOS CUIDADORES SOBRE AS PATOLOGIAS DE CRIANÇAS E ADOLESCENTES INTERNADOS E OS FATORES ASSOCIADOS

RESIDENTE: DANUSA DA PAZ OLIVEIRA DE SOUSA LEAL

PROGRAMA: PEDIATRIA

INSTITUIÇÃO:

ORIENTADOR: SIMONE SOARES LIMA

INTRODUÇÃO: A internação pode repercutir negativamente no desenvolvimento, interação social e no convívio familiar da criança e adolescente. O conhecimento sobre a doença pode reduzir o tempo de hospitalização, melhorar adesão ao tratamento e o prognóstico. OBJETIVO: Avaliar o conhecimento dos cuidadores de crianças e adolescentes internados sobre suas patologias e fatores associados. Identificar o perfil socioeconômico-demográfico do cuidador, clínico dos pacientes, qualidade e satisfação do atendimento. METODOLOGIA: Estudo transversal, quantitativo. Entrevistou-se cuidadores de crianças e adolescentes menores de dezesseis anos internados no Hospital Infantil Lucídio Portela sobre a condição socioeconômica-demográfica e educacional da família, dados clínicos e conhecimento sobre a doença e sobre a qualidade e satisfação com equipe multiprofissional. Utilizou-se testes bicaudais e nível de significância estatística a de 0,05. RESULTADOS: Foram entrevistados 64 cuidadores, $89,1 \%$ pais, escolaridade até ensino fundamental (50\%), procedência urbana $(60,9 \%)$, casados ou em união estável $(78,1 \%)$ e com renda até dois salários mínimos $(92,2 \%)$. As doenças tiveram evolução predominante entre 120 e 365 dias $(39,1 \%)$ e internação inferior a 30 dias $(54,7 \%)$ com as principais informações fornecidas por pediatra assistente. O conhecimento foi moderado $(6,22 \pm 2,37)$, relacionado a procedência urbana $(0,025)$, internação hospitalar inferior a trinta dias $(0,030)$ e solteirice $(0,001)$. Bom grau de satisfação e qualidade do atendimento, este não relacionado ao conhecimento. Equipe 
médica com maior satisfação e fisioterapia com melhor atendimento. CONCLUSÃO: O conhecimento de cuidadores é moderado, relacionado a procedência urbana, internação menor de um mês e solteirice. Há boa satisfação e qualidade assistencial, este não relacionado ao conhecimento.

PALAVRAS-CHAVE: Criança. Adolescente. Conhecimento. Hospitalização. Cuidadores.

TCC 32 - PERFIL DE ADOLESCENTES COM COMPORTAMENTOS AUTOLESIVOS EM ESCOLA PÚBLICA DE CAXIAS - MARANHÃO

\section{RESIDENTE: JOSÉ HERMÍNIO ROCHA MAGALHÃES SANTOS \\ PROGRAMA: PEDIATRIA \\ INSTITUIÇÃO: \\ ORIENTADOR: CATARINA FERNANDES PIRES}

INTRODUÇÃO: A adolescência é um período marcado por diversas transformações fisiológicas e psicossociais, e é nesta fase que pode surgir condutas patológicas, como o comportamento autolesivo, caracterizado como um conjunto de ações que produzem dano físico ao próprio indivíduo. OBJETIVO: Caracterizar o perfil de adolescentes que apresentam comportamento autolesivo matriculados em uma escola pública na cidade de Caxias - MA. MÉTODOS: Trata-se de estudo observacional, de corte transversal e abordagem quantitativa realizado com 334 adolescentes entre 13 e 19 anos de uma escola pública de Caxias - MA, por meio da aplicação de um questionário padronizado e validado para investigar dados sociodemográficos, escolares, estilo de vida e prevalência de comportamentos autolesivos. Os dados foram analisados no programa estatístico Stata/SE ${ }^{\circledR}$ versão 10.0 , considerando uma significância estatística de $5 \% \quad(p \leq 0,05)$. RESULTADOS: A prevalência de comportamentos autolesivos entre os adolescentes foi de $44,9 \%$, com predominância entre as meninas. O método de autolesão mais comum foi o autoflagelo $(26,4 \%)$. O sexo feminino, viver com outro familiar, estar cursando o $3^{\circ}$ ano do ensino médio, consumir cigarros e bebida alcóolica foram fatores associados ao comportamento autolesivo entre os adolescentes. CONCLUSÃO: A prevalência de comportamentos autolesivos entre os adolescentes investigados é alta, requerendo maior atenção para a saúde mental dessa população. Estudos bem delineados que forneçam uma compreensão mais clara das causas dos comportamentos autolesivos em adolescentes são necessários.

PALAVRAS-CHAVE: Adolescente. Conduta Autolesiva. Comportamento de Risco. Saúde do Adolescente.

TCC 33 - RELAÇÃO ENTRE TEMPO DE PERMANÊNCIA HOSPITALAR E BACTÉRIAS MULTIDROGA RESISTENTES EM HOSPITAL INFANTIL DE REFERÊNCIA

RESIDENTE: LAIS PORTELA NEIVA COELHO

PROGRAMA: PEDIATRIA

INSTITUIÇÃO:

ORIENTADOR: BRUNO PINHEIRO FALCÃO

INTRODUÇÃO: Infecção hospitalar $(\mathrm{IH})$ é definida como aquela adquirida após a admissão do paciente em um hospital. OBJETIVOS: Fornecer dados referentes às infecções hospitalares em pediatria, com enfoque na relação entre tempo de permanência hospitalar e bactérias multirresistentes. METODOLOGIA: A amostra foi composta por todos os pacientes internados em hospital infantil de referência, que evoluíram com $1 \mathrm{H}$, com resultado de culturas positivas, entre maio de 2017 e maio de 2018. A coleta de 
dados foi realizada com preenchimento de ficha elaborada pelos pesquisadores. RESULTADOS: A maioria dos participantes eram meninos $(61,27 \%)$, com idade entre um e cinco anos (36,76\%), tempo de internação entre dois e cinco meses e entre seis a doze meses (igual porcentagem, 30,88\%). A maioria dos pacientes eram procedentes da Unidade de Terapia Intensiva (UTI) (78,92\%) e 65,20\% encontravam-se em ventilação mecânica (VM). O acesso venoso central foi o dispositivo invasivo mais encontrado $(39,22 \%)$ e a urocultura, o sítio com maior índice de culturas positivas. A Klebsiella sp foi a bactéria mais encontrada nas culturas avaliadas, a Pseudomonas aeruginosa a mais encontrada na UTI e a Klebsiella sp, na enfermaria. O aumento do tempo de internação elevou de forma crescente o número de pacientes com multirresistência $(p<0,05)$. Os leitos de UTI apresentaram os maiores índices de multirresistência $(78,88 \%)(p<0,05)$, bem como a necessidade de VM $(p<0,05)$. CONCLUSÃO: Tais evidências apontaram relação entre tempo de internação hospitalar, internação em UTI e VM como fatores interferentes nas infecções causadas por bactérias multirresistentes na amostra estudada.

PALAVRAS-CHAVE: Infecção Hospitalar. Resistência Microbiana a Medicamentos. Pediatria.

TCC 34 - CUSTO DO TRATAMENTO MEDICAMENTOSO DO GLAUCOMA PARA PACIENTES ATENDIDOS EM UM AMBULATÓRIO PÚBLICO

RESIDENTE: GUILHERME HENRIQUE MENDES LEAL DE SOUSA MARTINS

PROGRAMA: OFTALMOLOGIA

INSTITUIÇÃO: HOSPITAL GETÚLIO VARGAS

ORIENTADOR: VITOR GOMES PRADO

INTRODUÇÃO: O glaucoma é a principal causa de cegueira permanente no mundo, apresentando-se na maioria dos casos com pressão intraocular elevada. $O$ tratamento inicial geralmente é feito com colírios, podendo o custo dessas medicações levar à interrupção do tratamento. OBJETIVO: Avaliar o custo do tratamento medicamentoso do glaucoma, o comprometimento da renda e suas associações com a interrupção do tratamento. METODOLOGIA: Aplicou-se questionário a 40 pacientes atendidos no Departamento de Oftalmologia do Ambulatório Integrado Dirceu Mendes Arcoverde Hospital Getúlio Vargas (HGV), Teresina-PI. Foram pesquisadas informações referentes a: nome, quantidade, custo mensal e forma de uso da medicação antiglaucomatosa; realização de cirurgia para tratamento do glaucoma; rendas pessoal e domiciliar; interrupção do tratamento do glaucoma; compra de outras medicações de uso contínuo e sua interrupção; opinião em relação ao preço das medicações e ao comprometimento de renda devido à compra delas. RESULTADOS: $O$ gasto mensal médio com medicação antiglaucomatosa foi $R \$ 75,11( \pm 67,05)$, equivalente a $7,16 \%( \pm 6,44)$ da renda pessoal e a $4,93 \%( \pm 5,06)$ da renda domiciliar. O gasto foi maior entre os participantes que faziam uso binocular da medicação $(p=0,049)$ e entre os que usavam mais de 01 tipo de colírio $(p=0,000)$. O comprometimento da renda pessoal foi maior entre os que já interromperam o seu tratamento devido ao gasto com colírios $(p=0,023)$. CONCLUSÃO: Verificou-se grande variabilidade no custo das medicações, gasto mais significativo com maior uso de colírios e que a interrupção do tratamento associa-se ao comprometimento da renda pessoal.

PALAVRAS-CHAVE: Glaucoma. Custos de Medicamentos. Renda. 
TCC 35 - ALTERAÇÕES OFTALMOLÓGICAS EM PORTADORES DE DOENÇA FALCIFORME EM HOSPITAL DE TERESINA

RESIDENTE: RAIMUNDO MOREIRA DOS SANTOS NETO

PROGRAMA: OFTALMOLOGIA

INSTITUIÇÃO: HOSPITAL UNIVERSITÁRIO DA UNIVERSIDADE FEDERAL DO PIAUÍ

ORIENTADOR: EDNALDO ATEM GONÇALVES E GILDENE ALVES DA COSTA

INTRODUÇÃO: A anemia falciforme (hemoglobinopatia genética autossômica recessiva) é a doença de caráter hereditário de maior prevalência no Brasil. No olho, manifesta-se através de alterações vaso-oclusivas, desde a órbita até a retina. OBJETIVO: Identificar e classificar possíveis alterações oftalmológicas em pacientes com doença falciforme acompanhados em serviços de referência em hematologia de Teresina. METODOLOGIA: Estudo descritivo transversal realizado no serviço de oftalmologia do Hospital Getúlio Vargas, com coleta de dados por questionário e exame oftalmológico de acuidade visual, biomicroscopia, tonometria e fundoscopia. Teste exato de Fisher foi aplicado para associação entre fundoscopia/biomicroscopia com o genótipo e uso de hidroxiureia. RESULTADOS: Avaliou-se 33 pacientes (66 olhos) com doença falciforme, sendo 18 do sexo feminino, com idade média de 13,4 anos. Mais de metade dos pacientes estava na faixa etária entre 10 e 12 anos $(51,52 \%)$ e se autodeterminaram pardos $(57,58 \%)$. Genótipo SS foi encontrado em 26 pacientes. A acuidade visual foi normal em quase todos os pacientes e 18 não referiram queixas. A biomicroscopia foi normal em 42 olhos, sendo as tortuosidades vasculares as principais alterações, presentes em 14 deles. A fundoscopia foi normal em $63,64 \%$ dos olhos. Retinopatia não-proliferativa estava presente em $15,15 \%$ dos olhos e em $3,03 \%$ a proliferativa. Tortuosidades vasculares foram observadas em $12,12 \%$. CONCLUSÃO: As principais alterações oculares encontradas à biomicroscopia foram as tortuosidades vasculares conjuntivais, e na fundoscopia, as tortuosidades vasculares, a retinopatia não-proliferativa (lesão black sunburst e pontos iridescentes) e a retinopatia proliferativa (lesão sea fan).

PALAVRAS-CHAVE: Anemia Falciforme. Olho. Microscopia com Lâmpada de Fenda. Fundo de Olho.

TCC 36 - ACHADOS CLÍNICOS E PATOLÓGICOS DE RETINOBLASTOMA EM HOSPITAL DE TERESINA, PIAUÍ

RESIDENTE: RICARDO COÊLHO FONTES

PROGRAMA: OFTALMOLOGIA

INSTITUIÇÃO:

ORIENTADOR: EDNALDO ATEM GONÇALVES

INTRODUÇÃO: O retinoblastoma é o tumor maligno intraocular primário mais comum em crianças, com mais de 7000 novos casos por ano no mundo(1). OBJETIVO: descrever os achados clínicos, demográficos e patológicos em pacientes com olhos enucleados por retinoblastoma. MÉTODOS: estudo observacional, com coleta de dados retrospectiva, baseado na análise de prontuários de pacientes enucleados por retinoblastoma no Hospital Getúlio Vargas no período de 2008 a 2018. RESULTADOS: Dos 41 pacientes, $58 \%$ eram do sexo masculino, $39 \%$ dos tumores eram bilaterais; Leucocoria foi o sinal inicial mais importante $(78,1 \%)$; Havia 2 pacientes $(4,9 \%)$ com história familiar. Todos os pacientes foram classificados nos estágios $D$ ou $E$ com base na Classificação Internacional do Retinoblastoma e submetidos a quimiorredução anterior à enucleação. A idade média de diagnóstico foi 27,07 meses, com um atraso médio de 6,43 meses em 
relação ao sintoma inicial. Todos os 6 pacientes $(14,6 \%)$ que apresentaram metástases foram a óbito. Foi encontrada associação estatisticamente significativa entre acometimento da coroide e casos de metástases $(p=0,017)$. Não foi observada associação entre grau de diferenciação, acometimento do nervo óptico pós-laminar, comprometimento da margem cirúrgica, esclera e segmento anterior com os casos de metástases. CONCLUSÃO: Os dados clínicos e demográficos foram semelhantes aos da literatura. $\mathrm{Na}$ análise dos dados histopatológicos, a presença de acometimento da coroide foi significativamente associada aos casos de metástases.

PALAVRAS-CHAVE: Retinoblastoma. Eye Cancer. Metastasis. Optic Nerve.

TCC 37 - IDEAÇÃO SUICIDA E FATORES ASSOCIADOS EM ESTUDANTES DE MEDICINA

RESIDENTE: JORDANO SAMPAIO GUIMARÃES SILVA

PROGRAMA: PSQUIATRIA

INSTITUIÇÃO:

ORIENTADOR: ALEXANDRE CASTELO BRANCO VAZ PARENTE

INTRODUÇÃO: o suicídio é o ato de causar intencionalmente a própria morte, associado a uma complexa interação biológica, psicológica, fatores socais, culturais e ambientais. Estima-se que mais de 800 mil pessoas morrem por suicídio anualmente no mundo. Os transtornos mentais são altamente prevalentes entre os estudantes universitários. E o reconhecimento de ideação suicida entre estudantes de medicina e seus fatores associados pode ajudar ao ser detectado em tempo precoce. OBJETIVOS: identificar o risco de suicídio em uma amostra de estudantes de medicina de uma universidade pública e correlacionar perfil socioeconômico, demográfico e acadêmico ao de ideação suicida na amostra estudada. METODOLOGIA: Estudo observacional descritivo com abordagem quantitativa e corte transversal com 126 estudantes que responderam a um questionário eletrônico com variáveis sociodemográficas e a Escala de Ideação Suicida e Desesperança de Beck (BSI e BHS). RESULTADOS: Em relação a ideação suicida, os dados obtidos mostram $69 \%$ (87) sem risco de cometer o ato suicida, $27,8 \%$ (35) com algum risco e $3,2 \%$ (4) com alto risco de suicídio. Em se tratando de desesperança, 51,6\% (65) com índice mínimo, 25,4\%(32) com índice leve, 16,7\%(21) índice moderado e $6,3 \%(8)$ índice grave. Conclusão: foi observado que o risco de suicídio avaliado no estudo se mostra semelhante a outros trabalhos na literatura.

PALAVRAS-CHAVE: Suicídio. Ideação Suicida. Estudantes de Medicina.

TCC 38 - USO DE SUBSTANCIAS PSICOATIVAS EM ACADÊMICOS DE MEDICINA RESIDENTE: ROBERTA SOARES GONDIM MEDEIROS FACUNDO PROGRAMA: PSIQUIATRIA

INSTITUIÇÃO: HOSPITAL UNIVERSITÁRIO DA UNIVERSIDADE FEDERAL DO PIAUÍ ORIENTADOR: LEONEL VELOSO SARAIVA

OBJETIVO: caracterizar o uso de substancias psicoativas em acadêmicos de medicina. METODOLOGIA: Trata-se de um estudo transversal, observacional, descritivo, com abordagem quantitativa, realizada com acadêmicos de Medicina. A coleta de dados sociodemográficos, como sexo, faixa etária, renda mensal, período do curso, tempo para lazer e prática de atividade física, foi realizada por meio de questionário. Para a investigação do uso de álcool, tabaco e outras drogas, utilizou o "Alcohol, Smoking and Substance Involvement Screening Test". $\mathrm{Na}$ análise dos dados recorreu-se à estatística 
descritiva, como frequências absolutas e relativas, para a inferencial, foi realizado o teste exato de Fisher. RESULTADOS: A maioria dos 126 acadêmicos era do sexo masculino $58,7 \%$, faixa etária entre 21 e 22 anos $92,9 \%$, renda mensal de três salários mínimos $68,3 \%$, entre o $5^{\circ}$ e 8 o período $46 \%$, sem tempo para lazer $70,6 \%$, e para a atividade física $63,5 \%$. O álcool foi a substancia psicoativa com maior frequência de uso habitual entre os estudantes de medicina, sendo seu uso 1 a 2 vezes por semana. Além do álcool, identificou-se o uso de outras substancias como o tabaco $27,8 \%$, maconha $27,0 \%$, Hipnóticos/Sedativos $18,3 \%$. Em relação a intervenção observou a necessidade de ser breve aos acadêmicos que faziam uso do álcool. CONCLUSÃO: evidencia-se por meio da análise dos resultados do estudo, que dentre as substancias psicoativas com maior frequência de uso entre acadêmicos de medicina, está o álcool. E, observa a necessidade de as instituições de ensino superior implementar estratégias de prevenção.

PALAVRAS-CHAVE: Estudantes de Medicina. Drogas. Drogas Ilícitas.

\section{AGRADECIMENTO}

UNIVERSIDADE FEDERAL DO PIAUÍ - UFPI 